\title{
Two-step activation of FOXO3 by AMPK generates a coherent feed-forward loop determining excitotoxic cell fate
}

\author{
D Davila ${ }^{1}$, NMC Connolly ${ }^{2}$, H Bonner ${ }^{1}$, P Weisová ${ }^{1,3}$, H Dussmann ${ }^{1,2}$, CG Concannon ${ }^{1,2}$, HJ Huber ${ }^{2}$ and JHM Prehn, ${ }^{\star, 2}$
}

Cerebral ischemia and excitotoxic injury induce transient or permanent bioenergetic failure, and may result in neuronal apoptosis or necrosis. We have previously shown that ATP depletion and activation of AMP-activated protein kinase (AMPK) during excitotoxic injury induces neuronal apoptosis by transcription of the pro-apoptotic BH3-only protein, Bim. AMPK, however, also exerts pro-survival functions in neurons. The molecular switches that determine these differential outcomes are not well understood. Using an approach combining biochemistry, single-cell imaging and computational modeling, we here demonstrate that excitotoxic injury activated the bim promoter in a FOXO3-dependent manner. The activation of AMPK reduced AKT activation, and led to dephosphorylation and nuclear translocation of FOXO3. Subsequent mutation studies indicated that bim gene activation during excitotoxic injury required direct FOXO3 phosphorylation by AMPK in the nucleus as a second activation step. Inhibition of this phosphorylation prevented Bim expression and protected neurons against excitotoxic and oxygen/glucose deprivation-induced injury. Systems analysis and computational modeling revealed that these two activation steps defined a coherent feed-forward loop; a network motif capable of filtering any effects of short-term AMPK activation on bim gene induction. This may prevent unwanted AMPK-mediated Bim expression and apoptosis during transient or physiological bioenergetic stress.

Cell Death and Differentiation (2012) 19, 1677-1688; doi:10.1038/cdd.2012.49; published online 27 April 2012

Excitotoxicity has been implicated in the pathogenesis of cerebral ischemia and several neurodegenerative disorders, ${ }^{1-3}$ and results from abnormal levels of the excitatory neurotransmitter glutamate (GLUT) in the synaptic cleft. ${ }^{4-6}$ The extent of ATP depletion during excitotoxic injury critically determines neuronal outcome. ${ }^{7}$ Neurons that fail to restore their ATP levels undergo rapid excitotoxic necrosis. ${ }^{7}$ However, many of the neurons that initially overcome the energetic crisis and recover their ATP levels nevertheless undergo a delayed apoptotic death. ${ }^{8}$ ATP depletion activates the AMPactivated protein kinase (AMPK), which adapts the cellular response to bioenergetic stress. ${ }^{9}$ AMPK switches off anabolic pathways, preventing ATP consumption, and switches on catabolic pathways, increasing glucose uptake and ATP production. ${ }^{10}$ AMPK-dependent plasma membrane translocation of the glucose transporter 3 , and subsequent glucose uptake mediate tolerance of neurons to excitotoxic or ischemic injury. ${ }^{11,12}$ In contrast to these cytoprotective mechanisms, however, prolonged AMPK activation has also been shown to contribute to ischemic injury and excitotoxicity. ${ }^{9,13,14}$ We recently demonstrated that prolonged activation of AMPK mediates excitotoxic apoptosis by inducing the expression of the pro-apoptotic Bcl-2 family protein Bim. ${ }^{13}$ However, the mechanisms that AMPK utilizes to switch on the transcriptional activation of bim during excitotoxic apoptosis remain unknown. Here, we describe the signaling pathways that couple AMPK activation to bim gene expression during excitotoxic apoptosis, and provide a mathematical framework that explains cell fate decision-making during AMPK activation.

\section{Results}

Bim induction during excitotoxic apoptosis requires AP-1 and FOXO3. We have previously shown that brief stimulation of NMDA receptors in cortical neurons or cerebellar granule neurons (CGNs) induces a bim-dependent, delayed excitotoxic cell death characteristic of caspaseindependent apoptosis. ${ }^{11,13,15}$ Western blot (Figure 1a) and qPCR analysis confirmed that CGNs exposed to GLUT/ glycine (GLY) $(100 / 10 \mu \mathrm{M})$ showed elevated Bim levels.

\footnotetext{
${ }^{1}$ Centre for the Study of Neurological Disorders, Royal College of Surgeons in Ireland, 123 St Stephen's Green, Dublin, Ireland and ${ }^{2}$ Department of Physiology and Medical Physics, Centre for Systems Medicine, Royal College of Surgeons in Ireland, 123 St Stephen's Green, Dublin, Ireland

*Corresponding author: JHM Prehn, Department of Physiology and Medical Physics, Royal College of Surgeons in Ireland, 123 St Stephen's Green, Dublin 2, Ireland. Tel: +353 1402 2261; Fax: +353 1402 2447; E-mail: prehn@ rcsi.ie

${ }^{3}$ Present address: Max F Perutz Laboratories, University of Vienna, Dr Bohr-Gasse 9, 1030 Vienna, Austria

Keywords: excitotoxicity; energy depletion; BH3-only protein BIM; FOXO3; computational modeling; network motifs

Abbreviations: AKT-CA, constitutively active mutant of AKT; AMPK-CA, constitutively active mutant of AMPK; CC, compound C; CFL, coherent feed-forward loop; CGNs, cerebellar granule neurons; $6 \times$ DBE FOXO3 promoter, reporter luciferase plasmid with six copies of the FOXO family protein-binding element; DN-FOXO3, dominant-negative FOXO3; FOXO3-6A, construct with FOXO3 sequence mutated at the AMPK phosphorylation sites; FOXO3-nuclear, construct with FOXO3 sequence mutated at the AKT phosphorylation sites; GLY, glycine; GLUT, glutamate; OGD, oxygen/glucose deprivation; PI, propidium iodide; RLUs, relative luciferase counts Received 30.9.11; revised 23.3.12; accepted 23.3.12; Edited by JM Hardwick; published online 27.4.12
} 


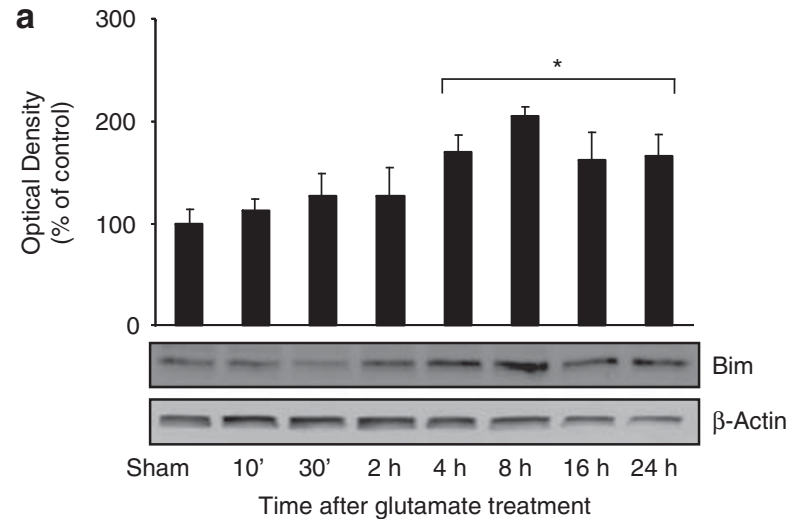

b
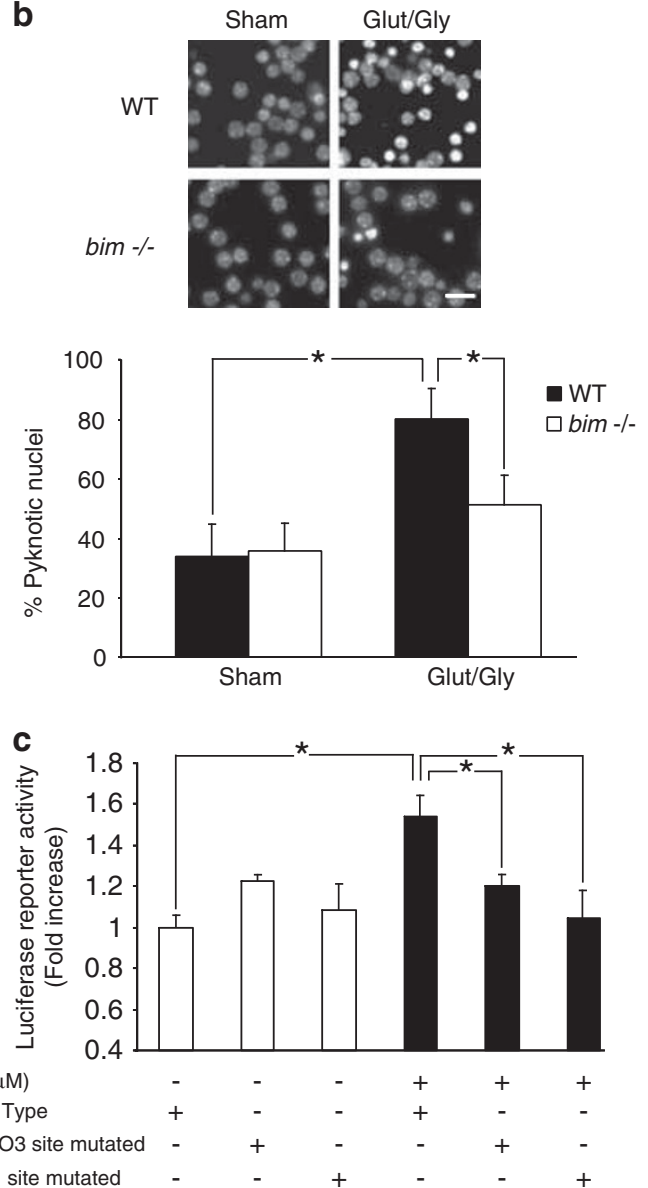

Glut / Gly (100/10 $\mu \mathrm{M})$ Bim promoter Wild Type Bim promoter $\mathrm{FOXO} 3$ site mutated Bim promoter AP-1 site mutated

Figure 1 Bim induction during excitotoxic apoptosis requires AP-1 and FOXO3. (a) Western blot analysis showed a significant increase in Bim levels within a 4-24 h time frame after GLUT/GLY $(100 \mu \mathrm{M} / 10 \mu \mathrm{M}, 30 \mathrm{~min})$ exposure $\left({ }^{*} P<0.05 ; n=5\right)$. $\beta$-actin served as loading control. (b) CGNs from bim - / - mice and wt controls were treated with GLUT or experimental buffer (sham conditions). $24 \mathrm{~h}$ after treatment the neurons were stained live with Hoechst and pyknotic nuclei scored $\left({ }^{*} P<0.05 ; n=3\right)$. Bar, $2.5 \mu \mathrm{m}$. (c) CGNs were transfected with a vector containing a $0.8 \mathrm{kB}$ fragment of the bim promoter. Bim promoter activation was significantly increased $24 \mathrm{~h}$ after GLUT exposure $\left({ }^{*} P<0.05 ; n=3\right)$. Mutations of the FOXO3 and AP- 1 binding sites significantly reduced this activation $\left({ }^{*} P<0.05 ; n=3\right.$ )

Excitotoxic injury was significantly reduced in CGNs deficient in bim (Figure 1b).

We next addressed the signaling pathways involved in bim activation during excitotoxic injury. Excellent candidates are the FOXO3 and AP-1 transcription factors, which have been implicated in Bim expression during neuronal apoptosis. ${ }^{13,16-18}$ We transfected CGNs with a reporter construct bearing the wild-type (wt) bim promoter sequence, or bim promoter constructs harboring mutations in the FOXO3 or AP-1 binding sites. Luciferase activity was significantly increased in GLUT-treated neurons expressing the wt bim promoter. However, this effect was abrogated by either bim promoter mutation (Figure 1c), indicating that FOXO3 and AP-1 binding sites were necessary for bim promoter activation.

AMPK-dependent downregulation of the mTOR/AKT pathway during excitotoxicity. Previously, we demonstrated that pAMPK activated JNKs, with JNK activation contributing to bim expression and apoptosis. ${ }^{13}$ We therefore next focused on exploring the role of $\mathrm{FOXO} 3$ activation in bim gene induction, and its control by AMPK. Firstly, we analyzed AMPK activation during excitotoxicity by western blot analysis of pAMPK $\alpha$ (Thr172) levels. AMPK activation was evident $10 \mathrm{~min}$ after GLUT exposure, and recovered to baseline levels after $120 \mathrm{~min}$ (Figure 2a).

The mTOR kinase complex (mTORC) is negatively regulated by AMPK. ${ }^{19}$ To address the regulation of $\mathrm{mTORC}$ during excitotoxicity, we monitored phospho-mTOR (Ser2448) levels (Figure 2b). GLUT treatment decreased p-mTOR levels $10 \mathrm{~min}$ after exposure, coinciding with the peak of AMPK activation. Notably, p-mTOR levels were persistently downregulated in response to GLUT. mTORC2 has been described as an important activator of the prosurvival kinase $A K T .^{20}$ We detected an early decrease in pAKT (Ser473) levels in response to GLUT (Figure 2c). In contrast to p-mTOR, however, pAKT levels partially recovered $2-8 \mathrm{~h}$ after GLUT exposure with complete recovery after $16 / 24 \mathrm{~h}$. The inactivation of AKT enables FOXO3-dependent gene transcription by preventing the cytosolic translocation of FOXO3. ${ }^{21}$ Western blot analysis of phospho-FOXO3 (Thr32) levels showed a significant decrease $2-4 \mathrm{~h}$ after GLUT exposure (Figure 2d).

To demonstrate that AMPK activation was responsible for the decrease in PAKT levels during excitotoxic injury, we depleted AMPK levels with vectors expressing an siRNA targeting AMPK- $\alpha 1 / \alpha 2$, or a control sequence ${ }^{13}$ (Supplementary Figure 1). CGNs with depleted AMPK levels presented significantly higher levels of phospho-AKT (Ser473) compared with control-transfected neurons (Figure 2e). This result was confirmed using the pharmacological inhibitor of AMPK, compound C (CC). CGNs pretreated with $\mathrm{CC}(10 \mu \mathrm{M}) 45 \mathrm{~min}$ before GLUT exposure presented with $69.5 \pm 19.5 \%$ higher phospho-AKT (Ser473) levels $30 \mathrm{~min}$ after GLUT treatment, compared with those pretreated with vehicle (data not shown). AMPK- $\alpha 1 / \alpha 2$ depletion also increased phospho-FOXO3 (Thr32) levels in neurons exposed to GLUT (Figure 2e). Confirming the downregulation of FOXO3 phosphorylation by AMPK, transfection of CGNs with a constitutively active mutant of AMPK (AMPK-CA) resulted in a decrease in phosphoFOXO3 (Thr32) levels and an increase in Bim expression (Supplementary Figures $2 \mathrm{~A}$ and B). Finally, to demonstrate that FOXO3 dephosphorylation was a consequence of AKT downregulation, we transfected CGNs with a constitutively 


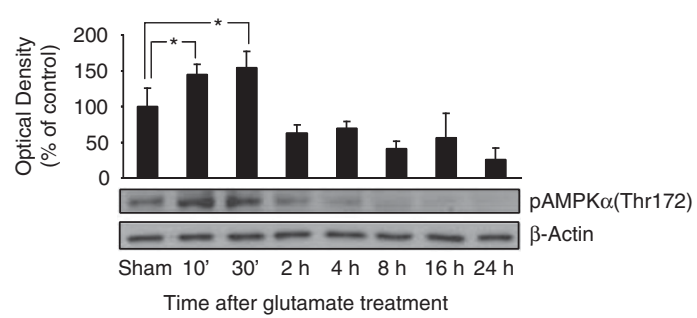

C

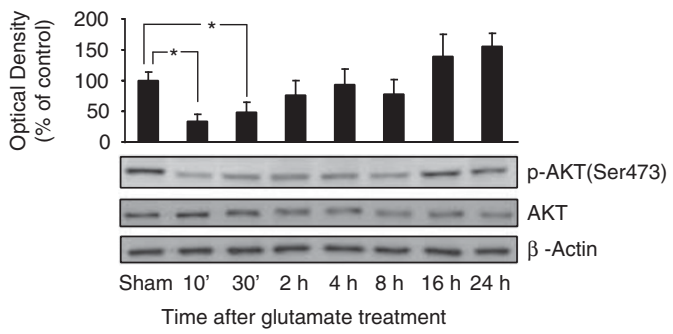

e

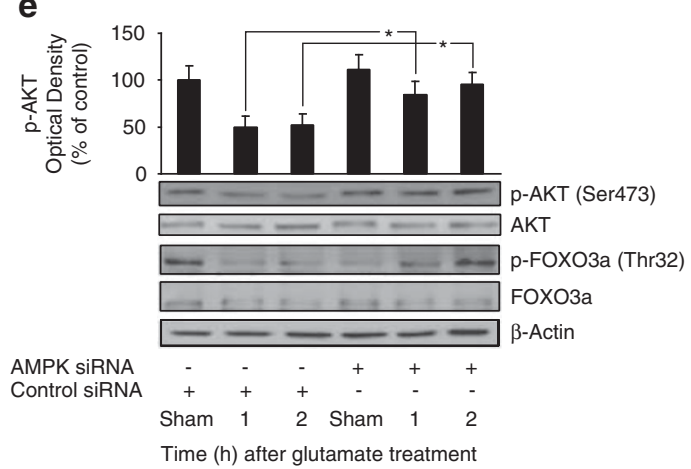

b

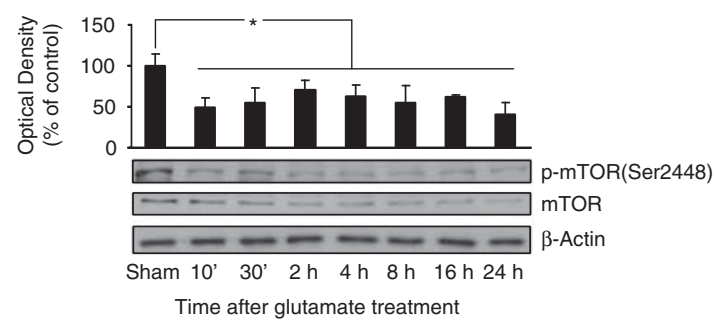

d

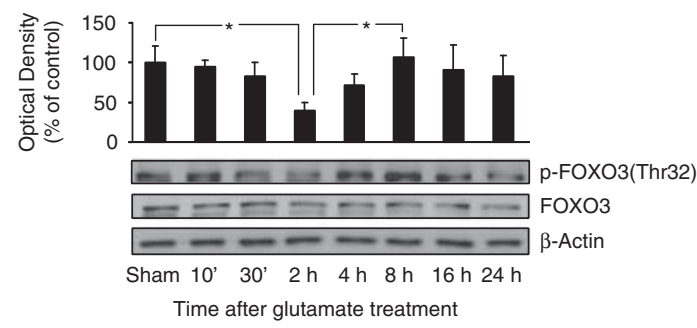

f

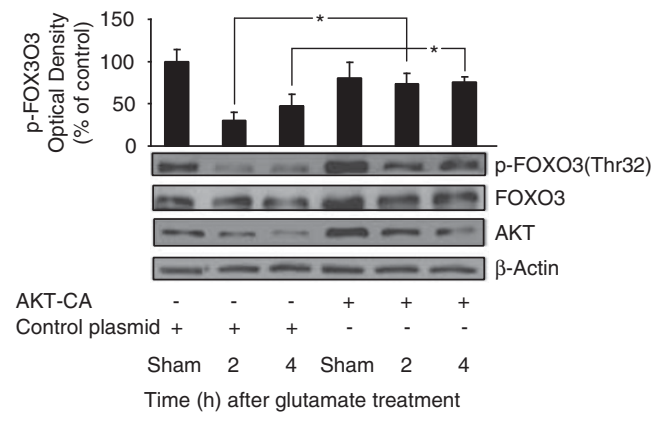

Figure 2 Downregulation of the mTOR/AKT pathway during excitotoxicity. (a) CGNs exposed to GLUT/ GLY (100/10 $\mu \mathrm{M}, 30 \mathrm{~min}$ ) increased phospho Thr172 AMPK $\alpha$ levels 10-30 min after exposure ( ${ }^{*} P<0.05 ; n=3$ ). $\beta$-actin served as loading control. (b) GLUT exposure permanently decreased phospho Ser2448 mTOR levels $\left({ }^{*} P<0.05\right.$; $n=3$ ). Total mTOR and $\beta$-actin served as loading controls. (c) GLUT reduced phospho Ser473 AKT levels 10 min- $2 \mathrm{~h}$ after GLUT exposure ${ }^{*} P<0.05 ; n=4$ ). Total AKT and $\beta$-actin served as loading controls. (d) GLUT reduced phospho Thr32 FOXO3 levels $2 \mathrm{~h}$ after GLUT exposure ( ${ }^{*} P<0.05 ; n=6$ ). Total FOXO3 and $\beta$-actin served as loading controls. (e) CGNs were transfected with AMPK siRNA or Control siRNA expressing vectors before GLUT exposure. Phospho Ser473 AKT/ Thr32 FOXO3 levels were analyzed ( 1 and $2 \mathrm{~h}$ ) after GLUT exposure. AMPK siRNA neurons presented higher levels than control siRNA neurons at both time-points $\left({ }^{*} P<0.05 ; n=3\right)$. Total AKT, FOXO3 and $\beta$-actin served as loading controls. AMPK depletion was monitored by quantification of total AMPK $\alpha$ levels (Supplementary Figure 1). (f) CGNs were transfected with AKT-CA or a control construct before GLUT exposure. AKT-CA neurons presented significantly higher phospho (Thr32) FOXO3 levels than control neurons ( 2 and $4 \mathrm{~h}$ ) after GLUT exposure ( $\left.{ }^{*} P<0.05 ; n=3\right)$. Total FOXO3 and $\beta$-actin served as loading controls. AKT-CA expression was monitored by total AKT levels

active mutant of AKT (AKT-CA). The expression of AKT-CA prevented the decrease in phospho-FOXO3 (Thr32) levels 2 and $4 \mathrm{~h}$ after GLUT exposure (Figure 2f).

AMPK activation during excitotoxicity induces FOXO3 nuclear translocation. We next analyzed whether the nuclear presence of $\mathrm{FOXO} 3$ was also increased in response to GLUT. CGNs were transfected with a FOXO3-GFP plasmid and exposed to either GLUT/GLY $(100 / 10 \mu \mathrm{M})$ or experimental buffer. Confocal images of single neurons were taken for FOXO3-GFP, Hoechst 33258 and propidium iodide (PI). Quantification of the GFP fluorescence intensity inside and outside the nucleus allowed the calculation of a nuclear/ cytoplasmic FOXO3-GFP ratio. In cells exposed to GLUT, $29.3 \pm 8.3 \%$ of neurons showed a sudden and permanent increase in this ratio, occurring with a delay that varied between 1 and $5 \mathrm{~h}$ after GLUT exposure (mean delay of $3 \mathrm{~h}$ $28 \pm 33 \mathrm{~min}$ ) (Figures $3 a$ and b). Of note, all neurons that exhibited this sudden and permanent increase underwent nuclear shrinkage and cell death $7-12 \mathrm{~h}$ after GLUT exposure (mean time-point $9 \mathrm{~h} 14 \pm 56 \mathrm{~min}$ ), as indicated by the loss of the GFP fluorescence signal and an increase in PI fluorescence. The remaining neurons that survived (62.8 $\pm 2.1 \%$ of the total population) did not show any change in their nuclear/cytoplasmic ratio, or showed a transient increase that recovered to baseline levels (mean duration of ratio increase $1 \mathrm{~h} 2 \pm 7 \mathrm{~min}$ ) (Supplementary Figures $3 \mathrm{~A}$ and $B$ ). Cells exposed to experimental buffer only did not show any alterations in the nuclear/cytoplasmic FOXO-GFP ratio (Supplementary Figure $3 \mathrm{C}$ ).

We investigated whether AMPK activation alone was sufficient to induce $\mathrm{FOXO} 3$ nuclear translocation. Expression of 


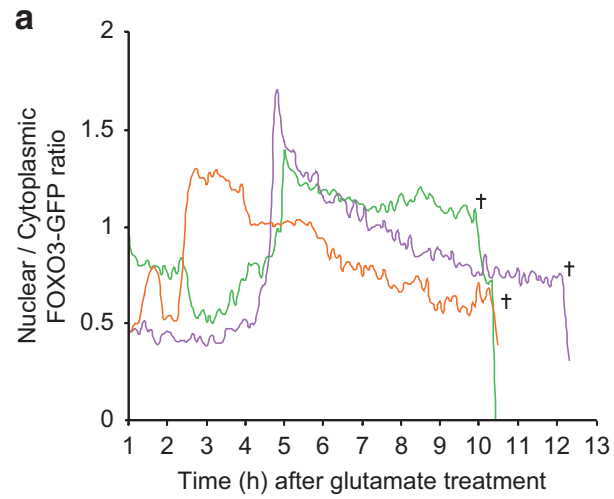

b
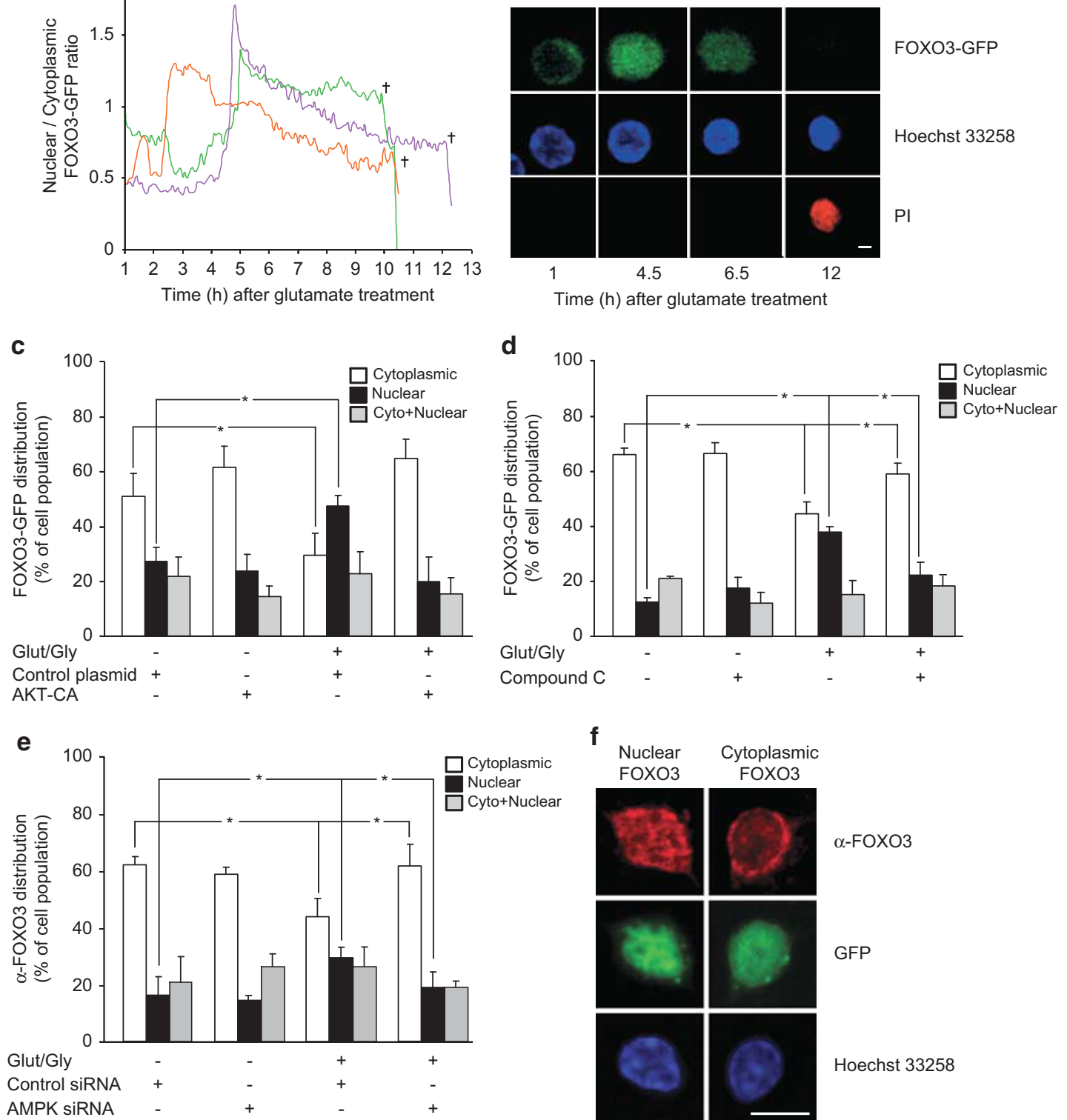

Figure 3 AMPK activation during excitotoxicity induces FOXO3 nuclear translocation. (a-d) CGNs were transfected with a FOXO3-GFP construct. (a) Traces of the nuclear/cytoplasmic FOXO3-GFP ratio in single CGNs as monitored using time-lapse microscopy. Following treatment with GLUT/ GLY (100/10 $\mu \mathrm{M})$ for $30 \mathrm{~min}, 29.3 \pm 8.3 \%$ of the neurons showed an increase in the nuclear/cytoplasmic FOXO3-GFP ratio, and its persistence was associated with a drop in GFP signal and neuronal death as indicated by PI uptake. Representative traces are shown. (b) Images extracted from the time-lapse series showing a CGN with FOXO3-GFP only in the cytoplasm at the start of the time series (1 h), persistent translocation of FOXO3-GFP to the nucleus $(4.5 \mathrm{~h})$, shrinkage of the nucleus $(6.5 \mathrm{~h})$, and subsequent secondary necrosis $(12 \mathrm{~h})$, bar, $10 \mu \mathrm{m}$. (c) FOXO3-GFP was co-expressed with the AKT-CA construct or a control vector. In the presence of the control vector, GLUT treatment increased the percentage of neurons with nuclear FOXO3-GFP (black bars) $3.5 \mathrm{~h}$ after exposure, and decreased the percentage with cytoplasmic FOXO3-GFP (white bars) ( ${ }^{\star} P<0.05 ; n=3$ ). AKT-CA expression prevented both effects. (d) CGNs were pretreated with CC $(10 \mu \mathrm{M})$ or vehicle for 45 min before GLUT exposure. CC pretreatment significantly reduced the increase in the percentage of cells with nuclear FOXO3-GFP (black bars), and the decrease in those with cytoplasmic FOXO3-GFP (white bars), $3.5 \mathrm{~h}$ after GLUT exposure $\left({ }^{\star} P<0.05\right.$; $n=3$ ). (e) CGNs were exposed to GLUT $48 \mathrm{~h}$ after transfection with AMPK siRNA or Control siRNA and were fixed and stained $3.5 \mathrm{~h}$ later with Hoechst and an antibody against FOXO3. AMPK siRNA significantly reduced the GLUT-induced increase in nuclear FOXO3 (black bars) and decrease in cytoplasmic FOXO3 (white bars), confirming the effects seen in (d) ( $\left.{ }^{*} P<0.05 ; n=4\right)$. (f) Images of single CGNs transfected with GFP-tagged siRNA and stained with an antibody against FOXO3 (red) and with Hoechst (blue), showing primarily nuclear (image series on left) or cytoplasmic (image series on right) FOXO3 staining. Bar, $5 \mu \mathrm{M}$

the AMPK-CA construct significantly increased the percentage of neurons showing FOXO3-GFP nuclear presence when compared with cells transfected with a control vector (Supplementary Figure 2C).
We next tested whether the nuclear translocation of FOXO3-GFP during excitotoxic injury was prevented either by expression of AKT-CA, or by inhibition of AMPK. We compared the percentage of cells exhibiting nuclear, 
cytoplasmic or cytoplasmic and nuclear presence of FOXO3GFP $3.5 \mathrm{~h}$ after GLUT or vehicle exposure. GLUT exposure significantly increased the percentage of neurons with nuclear FOXO3-GFP compared with control neurons, and decreased the percentage of neurons with cytoplasmic FOXO3-GFP (Figures $3 c$ and d). Both effects were abrogated by AKT-CA expression (Figure $3 e$ ) or by pretreatment with $\mathrm{CC}(10 \mu \mathrm{M})$ (Figure $3 \mathrm{~d})$. These results were confirmed with immunofluorescence experiments on neurons transfected with AMPK siRNA (Figures $3 e$ and f). These data demonstrated that excitotoxic injury induces FOXO3 nuclear translocation in an AMPK- and AKTdependent manner.

FOXO3 nuclear translocation is not sufficient for bim expression and cell death. In addition to FOXO3 nuclear translocation, further post-translational modifications may be necessary to stimulate its transcriptional activity. ${ }^{17,22}$ This hypothesis was tested by co-expressing the bim promoter plasmid with a FOXO3 construct, mutated at the AKT phosphorylation sites and therefore permanently localized to the nucleus ('FOXO3-nuclear'21). GLUT/GLY (100/10 $\mu \mathrm{M})$ exposure significantly increased luciferase activity in CGNs expressing 'FOXO3-nuclear' (Supplementary Figure 4A), suggesting that $\mathrm{FOXO} 3$ required further post-translational modifications to increase its binding to the bim promoter. Additional single-cell experiments implicated AMPK in mediating these events. Neurons transfected with FOXO3GFP were exposed to GLUT, and $2 \mathrm{~h}$ later treated with $\mathrm{CC}$ $(10 \mu \mathrm{M})$. We hypothesized that this treatment paradigm would avoid AMPK inhibition during or shortly after GLUT exposure, allowing FOXO3 nuclear translocation, but inhibiting AMPK subsequent to this. Indeed, late addition of $C C$ did not prevent FOXO3-GFP nuclear translocation. However, all neurons that displayed a permanent FOXO3 nuclear translocation failed to undergo subsequent nuclear shrinkage and cell death (Figures $4 a$ and $b$ ), and remained viable until termination of the experiments.

To corroborate these single-cell experiments we analyzed Bim and FOXO3 expression and localization by western blotting in CGNs treated with CC $2 \mathrm{~h}$ after GLUT exposure. CC-treated neurons expressed lower levels of Bim, without a decrease in FOXO3 nuclear translocation (Figures 4c and d). GLUT-induced cell death was also significantly impaired in this setting (Figure 4e). Taken together, this data suggest that AMPK may have a further essential role in bim activation that extends beyond inducing $\mathrm{FOXO} 3$ translocation, and that requires prolonged $\mathrm{AMPK}$ activity.

We also investigated $\mathrm{FOXO} 3$ translocation and Bim expression during varied durations of pharmacologicallyinduced AMPK activity. We found that although CGNs subjected to continuous AICAR treatment $(2.5 \mathrm{mM})$ showed increased Bim expression, no significant Bim induction occurred with transient addition of AICAR to the cultures for $1 \mathrm{~h}$ despite FOXO3 nuclear translocation in both treatment paradigms. Increased nuclear localization of pAMPK was also observed after continuous, but not transient, AICAR treatment (Figure 4f), confirming that prolonged AMPK activation is required for Bim expression, and suggesting that nuclear pAMPK may mediate this Bim expression.
FOXO3 activation by AMPK is a coherent feed-forward network motif that can act as a suppressor of transient stress signals. To understand the regulatory role of AMPK in FOXO3 activation and Bim expression from a systems point of view, we employed computational modeling. Initially, we modeled (by ordinary differential equations) a one-step FOXO3 activation where pAMPK-mediated Akt inhibition lead to $\mathrm{FOXO} 3$ dephosphorylation (FOXO3 dephos $_{\text {) and }}$ activated FOXO3 for Bim expression (Figure 5a, denoted by (1)). This 'linear' pathway predicted a similar FOXO3 activation, and therefore Bim expression, following both short-term and long-term periods of AMPK activity (Figure 5b). However, this linear model neither explained our results that the duration of AMPK activation may determine whether Bim is expressed or not (Figure 4f), nor did it support previous findings that short-term pAMPK activation was neuroprotective. ${ }^{11,23}$ We therefore assumed that an additional regulation step was required to prevent Bim expression following short-term AMPK activation, while maintaining robust Bim expression following prolonged AMPK activation.

We incorporated into our model a second activation step of

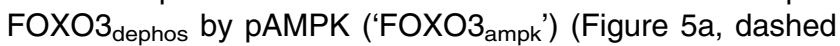
line denoted by (2)). In this model, Bim induction was suppressed for short-term pAMPK activity (Figure 5c, $40 \mathrm{~min}$ ), as the pAMPK signal (second activation step) was no longer active by the time the signal of the first activation step had induced FOXO3 dephosphorylation. Prolonged pAMPK activation, however, outlasted the delay of the first step and led to a pronounced increase in Bim expression (Figure 5c, $100 \mathrm{~min}$ ). This gave a qualitatively good explanation of our findings in Figure $4 \mathrm{f}$, where long-term AMPK activation led to significant Bim expression, whereas shortterm activation did not. The inclusion of a second FOXO3 activation step by pAMPK resulted in a network motif known as a coherent feed-forward loop (CFL).

AMPK induces bim expression by direct FOXO3 phosphorylation. AMPK has been shown to regulate the DNA binding of transcription factors by direct phosphorylation. ${ }^{10,24,25}$ Interestingly, in non-neuronal cells, AMPK has been shown to specifically activate $\mathrm{FOXO} 3$ by phosphorylation. $^{24}$ To validate the modeling results we tested if AMPK phosphorylation of $\mathrm{FOXO} 3$ was required for Bim expression. We co-expressed a FOXO3 construct (FOXO3-6A), which is mutated at the six residues (Thr179, Ser399, Ser413, Ser439, Ser555 and Ser588) phosphorylated by AMPK, alongside the bim promoter reporter plasmid. As expected, CGNs co-transfected with FOXO3 wt and treated with GLUT showed a significant increase in luciferase activity compared with control neurons. However, in neurons co-transfected with the FOXO3-6A mutant, this effect was abrogated (Figure 6a, upper panel). These results were also confirmed by western blot analysis of Bim protein levels (Figure 6a, lower panel).

Next we tested whether FOXO3 translocation during excitotoxicity also required $\mathrm{FOXO} 3$ phosphorylation by AMPK. CGNs were transfected with FOXO3-6A or FOXO3 wt constructs and then exposed to GLUT or experimental buffer. Three hours after exposure cultures were subjected to 
subcellular fractionation. GLUT treatment induced a similar increase in $\mathrm{FOXO} 3 \mathrm{wt}$ and FOXO3-6A nuclear levels when compared with control neurons (Figure 6b). A similar decrease was observed in cytosolic FOXO3 wt and FOXO3-6A levels (Flag M2-tag), suggesting that FOXO3 phosphorylation by AMPK did not affect FOXO3 translocation. We also observed that pAMPK $\alpha$ (Thr172) levels were increased in the nucleus and decreased in the cytosol after GLUT treatment (Figure 6b), suggesting the nuclear translocation of both $\mathrm{pAMPK}$ and FOXO3 during excitotoxicity. The

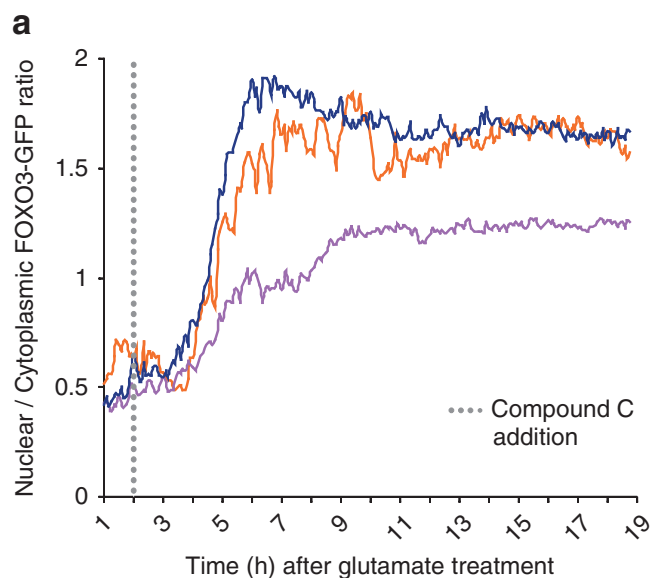

b
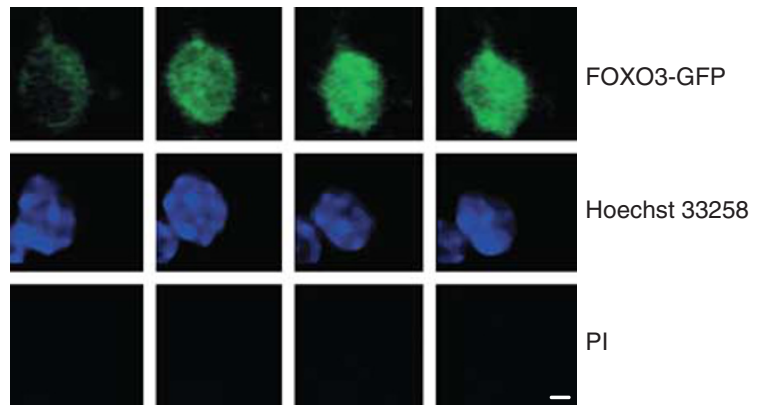

12

16

Time (h) after glutamate treatment

d

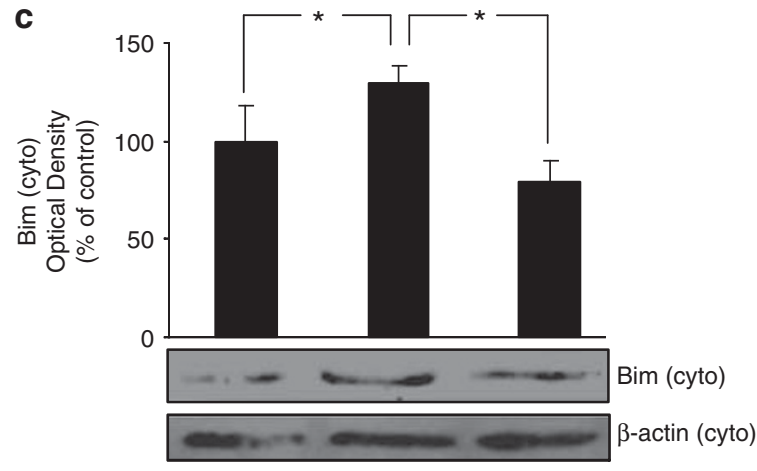

Compound C

(2 h after Glut)

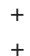

ater Glut)
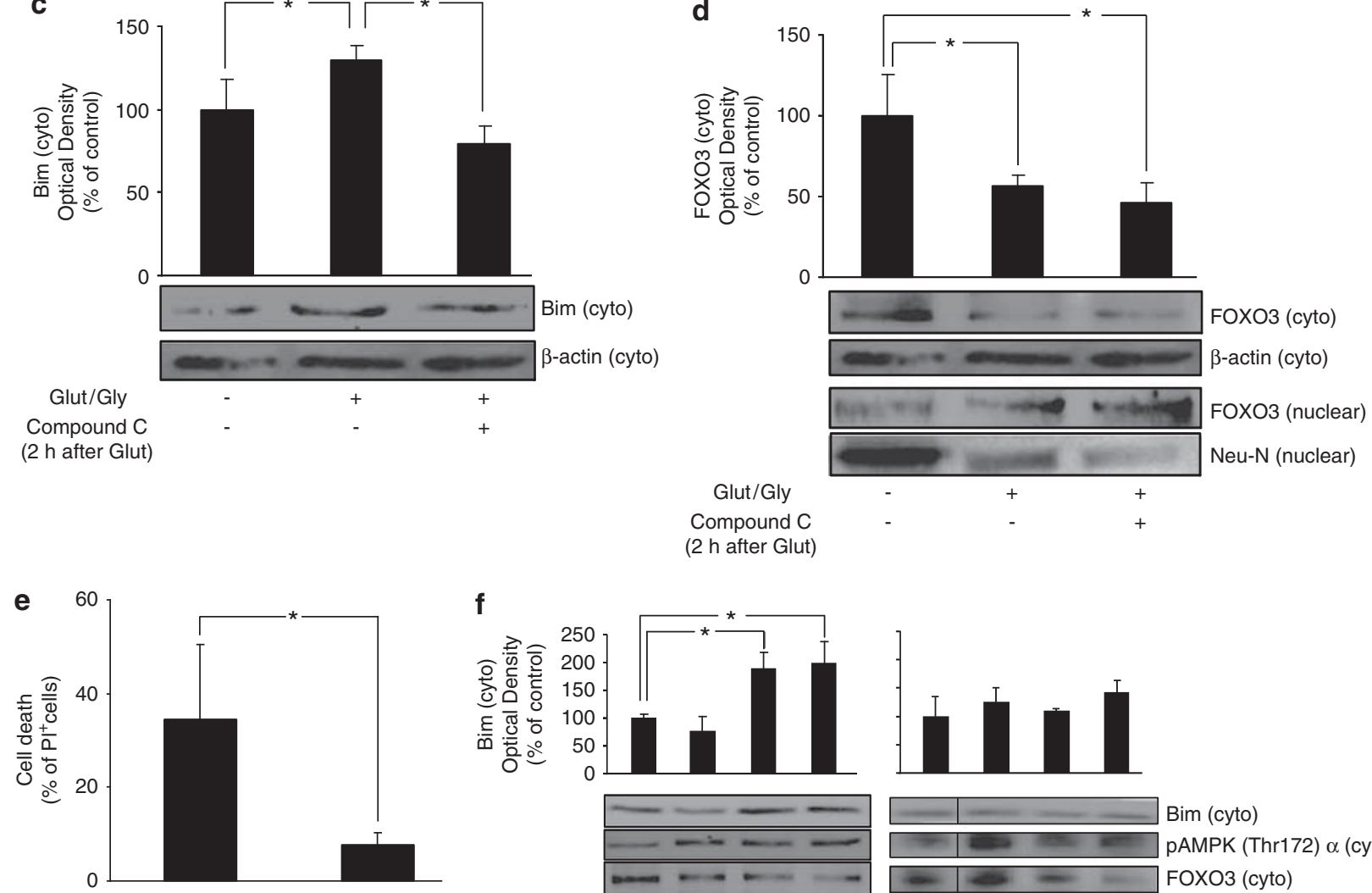

f

Glut/Gly

Compound C

(2 h after Glut)
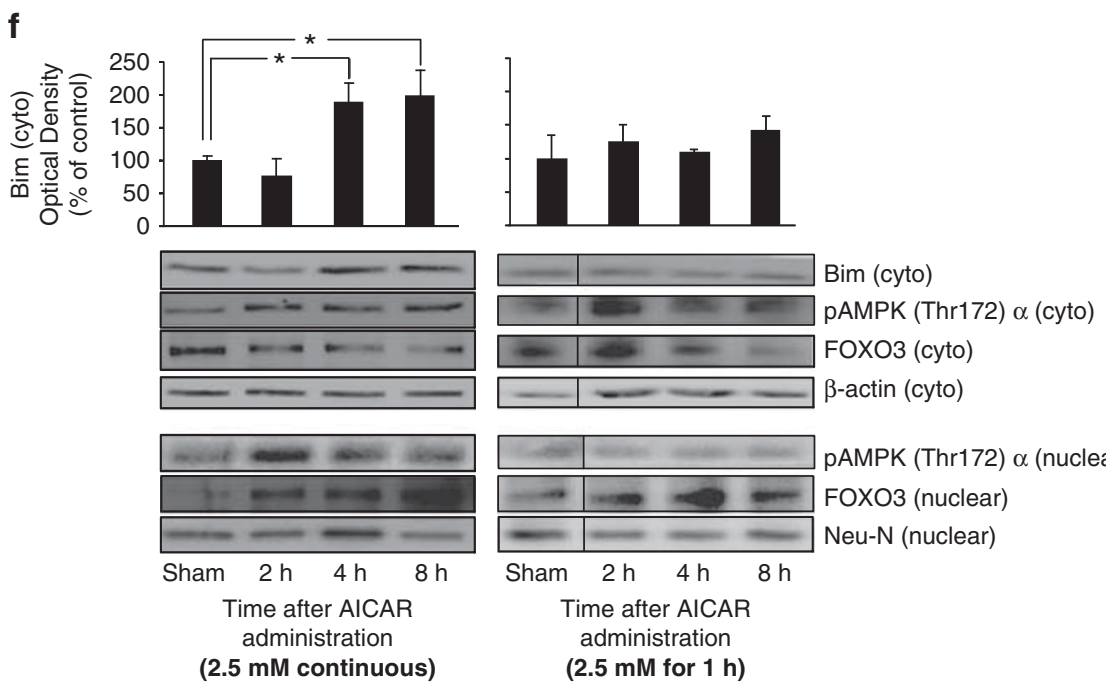

pAMPK (Thr172) $\alpha$ (nuclear)

FOXO3 (nuclear) Neu-N (nuclear)

(2.5 $\mathrm{mM}$ for $1 \mathrm{~h}$ ) 
fact that nuclear pAMPK is enriched $3 \mathrm{~h}$ after GLUT exposure indicates that FOXO3 phosphorylation by AMPK can occur subsequent to $\mathrm{FOXO} 3$ nuclear translocation. We next directly tested whether the phosphorylation state of FOXO3 in the nucleus depended on AMPK activity. CGNs transfected with the 'FOXO3-nuclear' mutant were pretreated with CC $(10 \mu \mathrm{M})$ and later exposed to GLUT. Four hours later, we detected elevated levels of 'FOXO3-nuclear' serine phosphorylation in neurons exposed to GLUT when compared with controls. This effect was abrogated in neurons pretreated with CC (Figure 6c).

The above results showed that $\mathrm{FOXO} 3$ phosphorylation by AMPK increased bim promoter activation during excitotoxic injury. This phosphorylation was specific to the bim promoter, as FOXO3-6A expression did not affect the activation of a chimeric promoter bearing six canonical FOXO-binding elements (TTGTTTAC box) not present in the bim promoter (Supplementary Figure 4B). We next tested if inhibition of FOXO3 phosphorylation by AMPK could facilitate the activation of alternative $\mathrm{FOXO} 3$ targets, such as the promoter of the stress tolerance gene $M n S O D .^{22}$ A FOXO3 wt or a FOXO3$6 \mathrm{~A}$ construct were co-expressed with a luciferase reporter plasmid containing the MnSOD promoter sequence (pSODluc) in CGNs. GLUT exposure increased luciferase activity in control neurons transfected with $\mathrm{FOXO} 3$ wt (Figure 6d). However, FOXO3-6A expression increased luciferase activity in neurons exposed to either experimental buffer or GLUT. This effect was abrogated by mutation of the FOXO3 binding sites in the MnSOD promoter (pSOD-luc-mut) (Figure 6d).

FOXO3 activation by AMPK is required for cell death. Next, we analyzed whether inhibition of FOXO3 phosphorylation by AMPK decreased neuronal injury during excitotoxicity (Figure 7a). We co-transfected CGNs with a GFPexpressing plasmid and either the $\mathrm{FOXO} 3 \mathrm{wt}$, dominantnegative $\mathrm{FOXO} 3$ (DN-FOXO3), or FOXO3-6A expression plasmid. Cell death was determined by evaluating the percentage of $\mathrm{PI}^{+}$and $\mathrm{GFP}^{+}$cells before and $16 \mathrm{~h}$ after GLUT/GLY $(100 / 10 \mu \mathrm{M})$ exposure. As shown in Figure 7a, FOXO3 wt-transfected neurons treated with GLUT showed significantly higher levels of cell death than neurons exposed to vehicle. However, cell death was negligible in cells transfected with DN-FOXO3 or FOXO3-6A.

To explore the possible relevance of $\mathrm{FOXO} 3$ regulation by AMPK in ischemic neuronal death, we subjected primary mouse cortical neurons to oxygen/glucose deprivation (OGD)-induced neuronal injury. Neurons were co-transfected with a GFP expression vector and either DN-FOXO3, or
FOXO3-6A expression constructs. Cultures were subjected to OGD for $30 \mathrm{~min}$ in a hypoxia chamber, and subsequently returned to normoxic/normoglycemic conditions for $24 \mathrm{~h}$. Cell death was determined by evaluating the percentage of pyknotic nuclei in the GFP ${ }^{+}$cells. As shown in Figure $7 \mathrm{~b}$, transfection with FOXO3-6A led to significantly lower levels of cell death when compared with cells co-transfected with a control plasmid, an effect that was comparable to the effect of DN-FOXO3.

\section{Discussion}

The present study describes the pro-apoptotic activity of the energy sensor AMPK during excitotoxic injury. Although AMPK activation has been associated with cell survival, ${ }^{11,23,26}$ it is now evident that it is also involved in longterm cell fate decisions. AMPK activation has been shown to activate apoptosis in different cell types, ${ }^{27,28}$ including neurons, ${ }^{9,13}$ and this apoptosis occurs after prolonged periods of AMPK activation. ${ }^{27,28}$ AMPK has also been shown to mediate ischemic neuronal death in vivo. ${ }^{14}$ As apoptosis is a terminal cellular decision, the signaling pathways controlling the pro-apoptotic activities of AMPK require a complex regulation. We here demonstrate that the combined activation of AP-1 and FOXO3 transcription factors by AMPK is required for the transcriptional activation of the pro-apoptotic bim gene. Indeed, bim activation and cell death induction required both AMPK-dependent FOXO3 nuclear translocation and direct FOXO3 phosphorylation by AMPK. Systems analysis and computational modeling showed that this two-step activation of FOXO3 provides a means for filtering out effects of shortterm AMPK activation on bim induction, while providing robust bim activation following prolonged AMPK activity.

Previous work from our group has demonstrated that AMPK mediates cell death by expression of the pro-apoptotic BH3only proteins $\mathrm{Bim}$ and, in some cell types, Bmf. ${ }^{13,29} \mathrm{Bim}$ promoter mutation of either the $\mathrm{FOXO} 3$ or the AP-1 binding site prevented bim activation in neurons during excitotoxicity. We demonstrate that in addition to the engagement of multiple transcription factors, individual transcription factors may also be subject to additional post-translational control steps in order to efficiently activate bim transcription. We observed that AMPK transiently inhibited AKT signaling during excitotoxicity, and that AKT-mediated FOXO3 dephosphorylation was a key step that allowed the nuclear translocation of FOXO3. ${ }^{21}$ This translocation was permanent in neurons, which underwent apoptosis, and was transient or did not occur in surviving cells. Specific mechanisms must therefore

Figure 4 FOXO3 nuclear translocation is not sufficient for bim expression and cell death. (a) Traces of the FOXO3-GFP ratio in single CGNs exposed to GLUT and $2 \mathrm{~h}$ later treated with $\mathrm{CC}(10 \mu \mathrm{M})$. In this case, all neurons that suffered a persistent FOXO3 nuclear translocation did not suffer subsequent nuclear shrinkage or cell death until termination of experiment. Representative traces are shown. (b) Images extracted from the time-lapse series showing a CGN with FOXO3-GFP in the cytoplasm at the start of the time series $(1 \mathrm{~h})$, persistent translocation of FOXO3-GFP to the nucleus $(6 \mathrm{~h})$, no shrinkage of the nucleus $(12 \mathrm{~h})$, and no subsequent secondary necrosis $(16 \mathrm{~h})$, bar, $10 \mu \mathrm{m}$. (c) CC $(10 \mu \mathrm{M})$ addition $2 \mathrm{~h}$ after GLUT exposure significantly reduced the GLUT-induced increase in Bim expression (measured $8 \mathrm{~h}$ after GLUT exposure, ${ }^{*} P<0.05$; $n=3)$. $\beta$-actin served as loading control. (d) CC $(10 \mu \mathrm{M})$ addition $2 \mathrm{~h}$ after GLUT exposure did not prevent the GLUT-induced decrease in cytoplasmic (cyto) FOXO3 or increase in nuclear FOXO3 levels (measured $8 \mathrm{~h}$ after GLUT exposure, $\left.{ }^{*} P<0.05 ; n=4\right)$. $\beta$-actin and Neu-N served as loading controls. (e) Addition of CC (10 $\left.\mu \mathrm{M}\right) 2 \mathrm{~h}$ after GLUT treatment significantly reduced the $\%$ of $\mathrm{PI}^{+}$cells detected $16 \mathrm{~h}$ after the excitotoxic insult $\left({ }^{\star} P<0.05 ; n=3\right)$. (f) CGNs exposed to continuous AICAR (2.5 mM) treatment showed an upregulation of Bim levels in the cytosol, and PAMPK (Thr172) $\alpha$ and FOXO3 levels in the nucleus $\left({ }^{*} P<0.05 ; n=3\right.$ ). CGNs exposed to transient AICAR treatment $(2.5 \mathrm{mM}, 1 \mathrm{~h}$, then washout) also showed increased nuclear FOXO3 levels; however, Bim levels in the cytosol and pAMPK in the nucleus were not upregulated $(n=3)$. $\beta$-actin and Neu-N served as loading controls. Times $(\mathrm{h})$ indicate duration of AICAR treatment (continuous) or recovery times following washout of AICAR 
Model Incorporating Coherent Feed-Forward Loop (CFL) Two-step FOXO3 activation

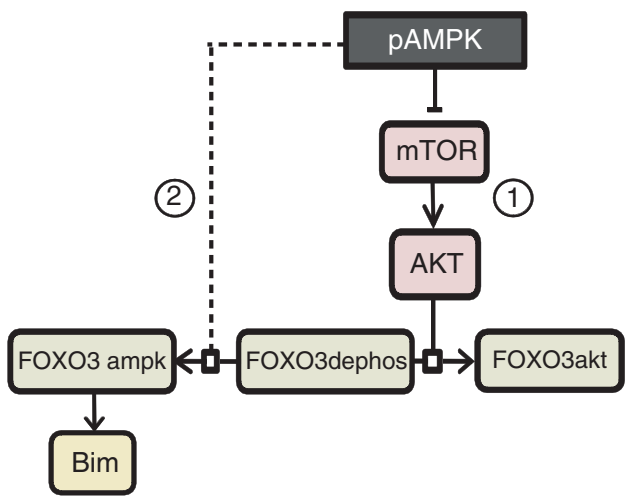

b

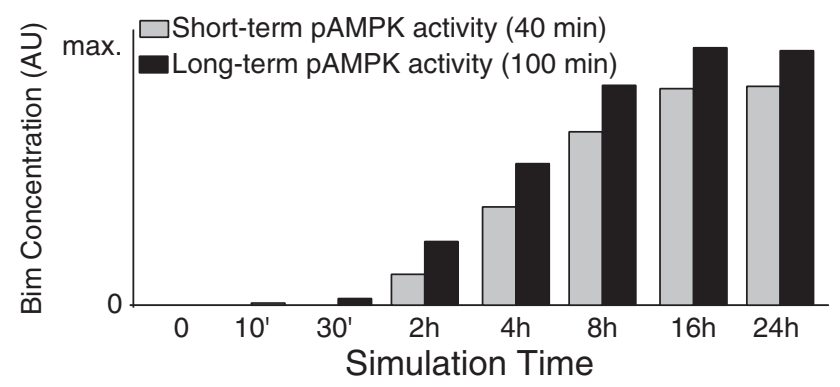

C

Model Incorporating CFL [1)+(2)]

Predicted Bim Expression

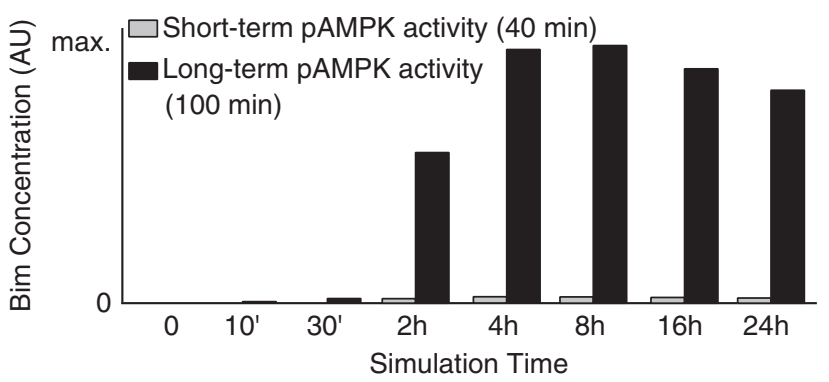

Figure 5 FOXO3 activation by AMPK is a coherent feed-forward network motif that can act as a suppressor of transient stress signals. A second activation step of FOXO3 is necessary to explain neuronal tolerance to short-term pAMPK activity while maintaining Bim expression following prolonged pAMPK activity. (a) pAMPKmediated FOXO3 activation was initially modeled by a linear pathway (denoted by (1)). (b) This linear pathway predicted similar Bim expression patterns following short- and long-term periods of pAMPK activity and conflicted with recent findings of the neuroprotective role of pAMPK. ${ }^{11}$ (c) Incorporation of the second activation step (denoted by (2) in (a)) reduced Bim expression following short-term pAMPK activity (40 min, gray bars). This network structure represents a CFL and, by preventing Bim expression in times of short-term stress, may allow PAMPK to exert its pro-survival effects. Significant Bim expression is still present following long-term pAMPK activity (100 min, black bars). Bars indicate relative differences in expression levels between both scenarios

exist to restore $\mathrm{pAKT} / \mathrm{pFOXO} 3$ levels in surviving neurons. Survival responses during excitotoxicity have been associated with enhanced neuronal glucose uptake and ATP availability, as well as with growth factor signaling, ${ }^{9,12}$ suggesting that these factors could restore $\mathrm{pAKT} / \mathrm{pFOXO3}$ levels. The persistent mTOR dephosphorylation after GLUT exposure suggests the involvement in this process of other AKT regulators besides mTOR. Given the central role of AKT in several cell survival pathways such as PI3K and GSK-3 $\beta$ signaling, ${ }^{30}$ the regulation of AKT independently from mTOR and AMPK pathways is not surprising and may involve phosphatases such as PP2A or PTEN. ${ }^{31,32}$

The AKT-dependent nuclear translocation of FOXO3, however, was not the sole determinant of FOXO3-dependent bim gene expression. Both biochemical and single-cell imaging experiments, as well as experiments using FOXO3$6 \mathrm{~A}$, demonstrated that bim activation and cell death additionally required FOXO3 phosphorylation by AMPK. In contrast, the transcription of the $M n S O D$ promoter was stimulated by FOXO3-6A expression alone. This finding suggested that direct phosphorylation by AMPK could guide $\mathrm{FOXO} 3$ towards its binding to the bim promoter instead of towards others targets such as the MnSOD promoter.

The two-step activation of FOXO3 by AMPK is a CFL network motif. Well established in systems biology, and overrepresented in pathways of gene regulation, CFLs are known to induce gene expression upon sustained, but not transient signals. ${ }^{33,34}$ Indeed, our identified CFL enables the filtering of transient AMPK impulses so that shorter periods of AMPK activity do not lead to Bim induction and allow pAMPK to exert its pro-survival effects. ${ }^{11}$ This filtering is achieved by the delay required for the AKT-mediated $\mathrm{FOXO} 3$ dephosphorylation in the first activation step. The period of AMPK activity must outlast this delay to activate $\mathrm{FOXO} 3$ in the second activation step. Increased survival signaling, through AKT activation, would prolong the delay of the first activation step, requiring a longer period of AMPK activity to induce Bim expression. This idea is supported by evidence showing a reduction in apoptosis following stimulation of AKT. ${ }^{35}$ Consequently, the $\mathrm{CFL}$ of $\mathrm{FOXO} 3$ activation may regulate Bim expression based on both stress duration and the extent of pro-survival signaling.

Previously, we demonstrated the involvement of JNK in Bim expression and the nuclear translocation of C-JUN induced by AMPK during excitotoxicity. ${ }^{13}$ Neurons have high levels of constitutive JNK1 activity, ${ }^{36}$ so it remains to be shown whether any surplus activation of JNK by AMPK contributes to bim gene induction during excitotoxic apoptosis. Such a potential third activation step by AMPK cannot be excluded, and may integrate other stress-related signals to form a more complex cascade of CFLs. Oxidative stress, for example, may activate the JNK/AP-1 pathway. ${ }^{36}$ Similarly, we cannot exclude a role of other transcription factors, such as Myb, ${ }^{18}$ in bim activation. Additionally, the FOXO family of transcription factors may be subject to other post-translational modifications such as JNK phosphorylation or SIRT1 de-acetylation. ${ }^{22,37}$

AMPK has previously been shown to control the DNAbinding of different transcription factors or co-factors by phosphorylation. ${ }^{10,24,25}$ AMPK phosphorylation of FOXO3 was not required for its nuclear translocation (see also Greer et al. ${ }^{24}$ ). However, it is possible that FOXO3 dephosphorylation and its subsequent nuclear translocation is a requirement for subsequent post-translational modifications (that is, FOXO3 phosphorylation by AMPK). ${ }^{17}$ Indeed, this is a 


\section{}

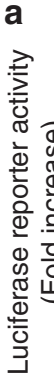

Glut/Gly $-\quad+$ FOXO3 $6 \mathrm{~A}$

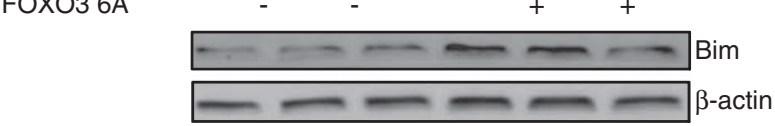

Glut/Gly Empty vector FOXO3 wild type FOXO3 $6 \mathrm{~A}$

\section{C}

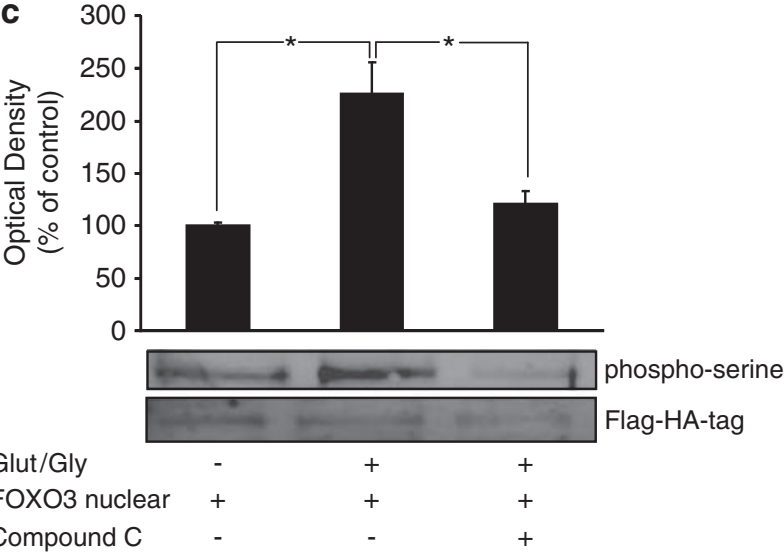

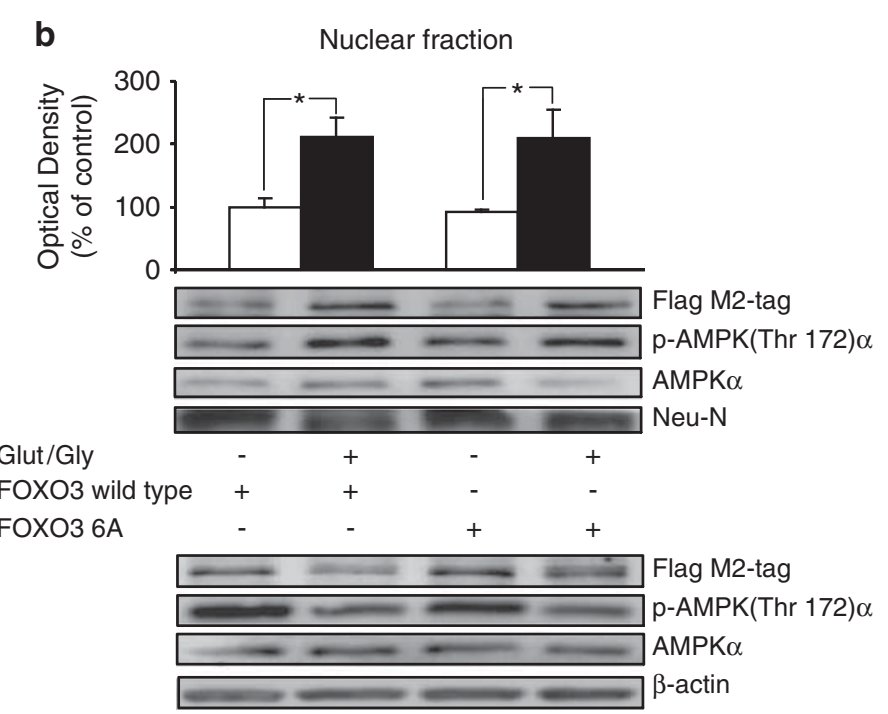

Cytosolic fraction

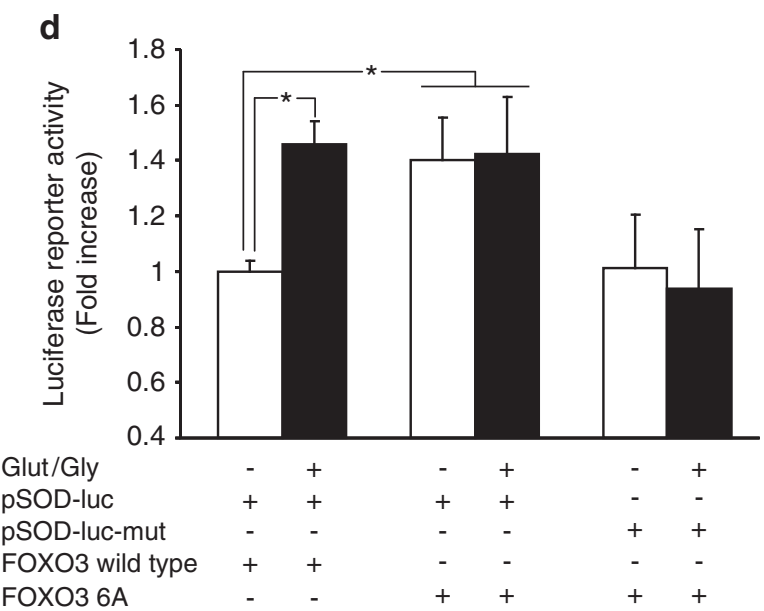

Figure 6 AMPK induces bim expression by direct FOXO3 phosphorylation. (a) Upper. CGNs were co-transfected with a vector containing the bim promoter and either the wt FOXO3 or FOXO3-6A constructs. GLUT/GLY $(100 / 10 \mu \mathrm{M}, 30 \mathrm{~min})$ exposure significantly increased bim promoter activation in cells transfected with FOXO3 wt $\left({ }^{*} P<0.05 ; n=4\right)$. FOXO3-6A expression prevented this effect $\left({ }^{*} P<0.05 ; n=4\right)$. (a) Lower. Western blot analysis confirmed the effect of FOXO3-6A on Bim protein levels after GLUT exposure (30 min). Results are representative of two independent experiments. (b) Upper. Nuclear fraction of CGNs transfected with FOXO3-Flag wt or FOXO3-6A-Flag constructs. Flag-tag detection by western blot and densitometry analysis showed a similar upregulation of both constructs $4 \mathrm{~h}$ after GLUT exposure $\left({ }^{\star} P<0.05 ; n=4\right)$. Nuclear phospho (Thr172) AMPK $\alpha$ levels were also upregulated. Neu-N was used as nuclear marker and $\beta$-actin as loading control. (b) Lower. GLUT exposure downregulated levels of FOXO3 wt and FOXO3-6A. Cytoplasmic phospho (Thr172) AMPK $\alpha$ levels were also downregulated. $\beta$-actin served as loading control. Representative blots are shown. (c) CGNs transfected with a 'FOXO3-nuclear' mutant (tagged with a flag-HA) and immunoprecipated showed an increase in Ser-phosphorylation $4 \mathrm{~h}$ after GLUT exposure $\left({ }^{*} P<0.05 ; n=3\right)$. Pre-treatment with $\mathrm{CC}(10 \mu \mathrm{M})$ for 45 min abrogated this effect $\left({ }^{*} P<0.05 ; n=3\right)$. (d) CGNs were co-transfected with a luciferase reporter containing a MnSOD promoter sequence (pSOD-luc) and either wt FOXO3 or FOXO3-6A. GLUT exposure increased luciferase activity in cells transfected with FOXO3 wt. However, FOXO3-6A expression increased luciferase activity in neurons exposed to experimental buffer or GLUT. This effect was abrogated by mutation of the FOXO3 binding sites in the MnSOD promoter (pSOD-luc-mut) $\left({ }^{\star} P<0.05 ; n=4\right.$ )

necessary condition for the functioning of the proposed CFL. We provided evidence of nuclear effects of AMPK on FOXO3 with Bim induction only detected after sustained AMPK activation and nuclear presence of PAMPK $\alpha$. Shorter periods of AMPK activity did not lead to AMPK translocation or Bim expression, although FOXO3 nuclear translocation was maintained in both scenarios. This evidence suggested that interaction of FOXO3 and AMPK in the nucleus could be a requirement for bim promoter activation and apoptosis. Interestingly, neuronal apoptosis was recently described in a model of Huntington's disease as dependent on nuclear accumulation of AMPK- $\alpha 1$. $^{38}$

In conclusion, our data suggest that $\mathrm{FOXO} 3$ activation represents a key step during bim-mediated excitotoxic neuronal death. The complex interplay between cellular bioenergetics, AMPK activation, and mTOR/AKT/FOXO3 signaling provides a molecular framework for cell fate decision making, preventing unwanted apoptosis activation during physiological AMPK activation or during conditions of mild bioenergetic stress. 
Materials and Methods

Materials. Fetal calf serum and minimal essential medium were obtained from Invitrogen (Bio Sciences, Dublin, Ireland). GLUT, GLY, Hoechst 33258 and PI were from Sigma-Aldrich (Arklow, Ireland). AICAR was from Cell Signaling (Isis Ltd, Bray, Ireland). CC was obtained from Calbiochem (Merck Biosciences, Nottingham, UK).

Preparation of primary CGNs. Both mouse (C57BL) or rat (SpragueDawley) cerebella were isolated from postnatal day 7 pups. CGN cultures were obtained as described previously. ${ }^{7}$ Cells were plated on poly-Lysine-coated glass

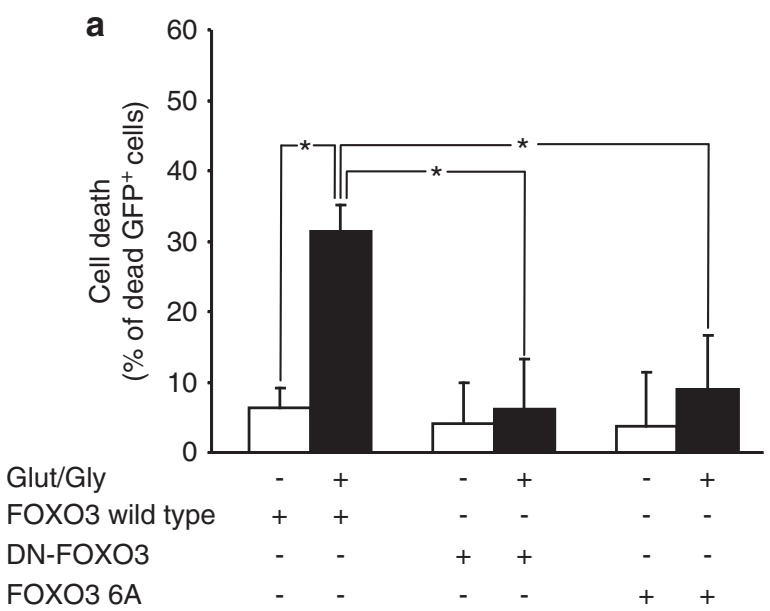

b
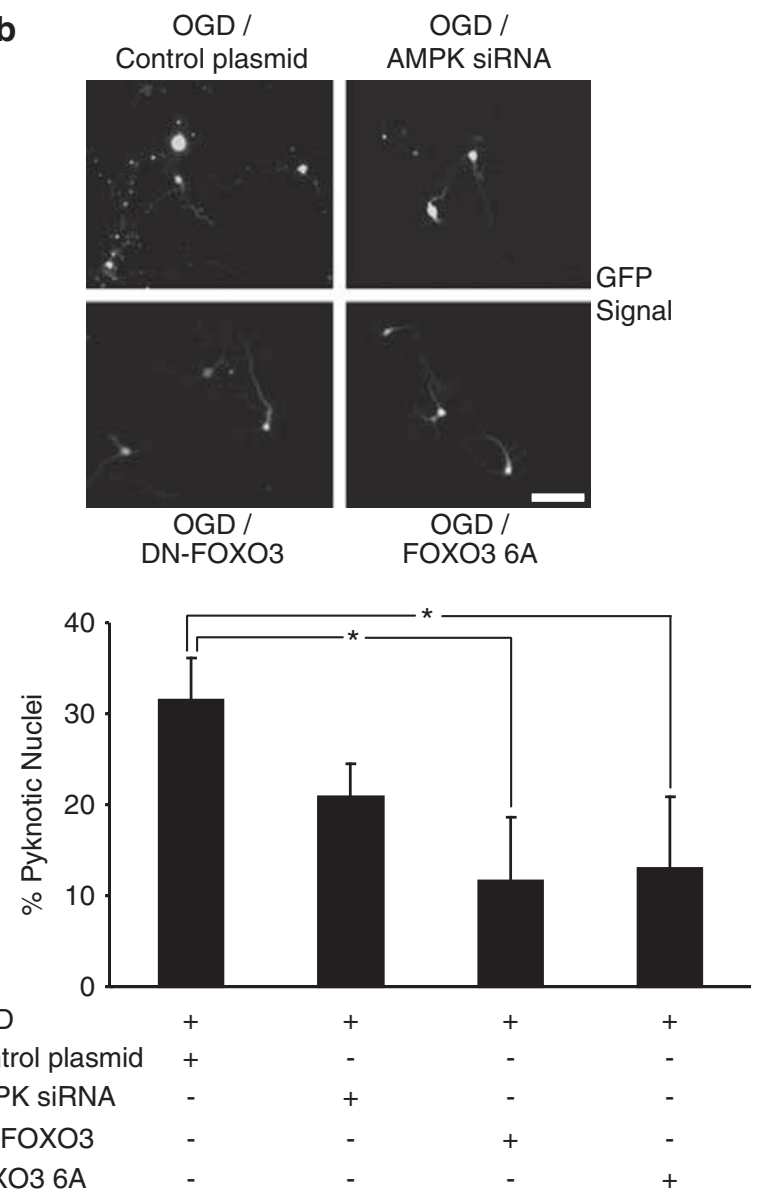

coverslips, glass Willco dishes, 6-well plates, and 24-well plates at $1 \times 10^{6} \mathrm{cell} / \mathrm{s} / \mathrm{ml}$, and maintained at $37^{\circ} \mathrm{C}$ in a humidified atmosphere of $5 \% \quad \mathrm{CO}_{2} / 95 \%$ air. Experiments were performed after 8-10 days in vitro. All animal work was performed with ethics approval from the RCSI and under licenses granted to the authors by the Irish Department of Health and Children.

Plasmids, siRNA and transfection. The FOXO3-GFP plasmid in an EGFP-N1 vector backbone was kindly provided by MP Smidt (University Medical Center, Utrecht, The Netherlands). The AMPK-CA construct is the truncated version of AMPK $\alpha 1$ after residue 312 and includes a Myc tag and was a kind gift from David Carling (Imperial College London, London, UK). The AKT-CA construct expresses the myristoylated AKT-1 sequence and was kindly provided by S Pons (Biomedicine Institute, CSIC, Barcelona, Spain). The Firefly luciferase reporter plasmid containing $0.8 \mathrm{kB}$ of the bim promoter sequence along with similar constructs with mutated $\mathrm{FOXO} 3$ or $\mathrm{AP}-1$ binding sites were kindly provided by Eric Lam (Imperial College London, London, UK). The pECE-FOXO3-TM (termed here 'FOXO3-nuclear'), a construct mutated at the three sites (T32A/S253A/S315A) of AKT phosphorylation and permanently located in the nucleus, was kindly provided by ME Greenberg (Harvard Medical School, Boston, MA, USA). The pECEFOXO3-M2-flag and pECE-FOXO3-6A-M2-flag (sextuple mutant T179A/S339A/ S413A/S555A/S588A/S626A at the sites for AMPK phosphorylation) were kind gifts from A Brunet (Harvard Medical School). The pmax-GFP construct was obtained from LONZA (Basel, Switzerland). The pRL-TK (TK Renilla luciferase), the $6 \times$ DBE FOXO3 (reporter luciferase plasmid with six copies of the FOXO family protein-binding element) and the reporter plasmids containing the MnSOD promoter sequence (pSOD-luc) along with a similar construct with mutated FOXO3 binding sites (pSOD-luc-mut) were a kind gift from BM Burgering (University Medical Center, Utrecht, The Netherlands). The DN-FOXO3 expresses the DNA-binding domain (amino acids 141-268) of pECE-FOXO3-TM, and was kindly provided by I Torres-Aleman (Cajal Institute, CSIC, Madrid, Spain). siRNA targeting AMPK- $\alpha 1 / \alpha 2$ (pFIV-AMPK-siRNA) and the control sequence (pFIV-Control-siRNA) were previously described. ${ }^{11}$ CGNs were transfected using calcium phosphate as previously described, ${ }^{11}$ the transfection reagent Neurofect from Genlantis (AMS Biotechnology, Milton, UK), or an electroporation kit (LONZA) as per manufacturer's instructions.

Live cell microscopy. CGNs on glass Willco dishes (WillCo Wells BV, Amsterdam, The Netherlands) were transfected with a FOXO3-GFP construct. After $48 \mathrm{~h}$ cells were treated for 30 min with GLUT and GLY $(100 \mu \mathrm{M}$ and $10 \mu \mathrm{M})$ in experimental buffer $\left(120 \mathrm{mM} \mathrm{NaCl}, 3.5 \mathrm{mM} \mathrm{KCl}, 0.4 \mathrm{mM} \mathrm{KH_{2 }} \mathrm{PO}_{4}, 20 \mathrm{mM}\right.$ HEPES, $5 \mathrm{mM} \mathrm{NaHCO}_{3}, 1.2 \mathrm{mM} \mathrm{Na}_{2} \mathrm{SO}_{4}, 1.2 \mathrm{mM} \mathrm{CaCl}_{2}, 1.2 \mathrm{mM} \mathrm{MgCl}_{2}$ and $15 \mathrm{mM}$ glucose; $\mathrm{pH}$ 7.4). Control CGNs were treated with experimental buffer only. Next, CGNs were stained with Hoechst 33258 and PI at a final concentration of $0.1 \mu \mathrm{g} / \mathrm{ml}$. Following treatment, the pre-conditioned medium, supplemented with HEPES (final concentration $20 \mathrm{mM}$ ), was replaced onto the cells and the dish was placed on the stage of a confocal microscope equipped with a $63 \times 1.4 \mathrm{NA}$ oil immersion objective and a thermostatically regulated chamber at $37^{\circ} \mathrm{C}$ (LSM 710 , Carl Zeiss, Jena, Germany). A thin layer of mineral oil $(1.5 \mathrm{ml})$ was added onto the medium to prevent evaporation. GFP was excited at $488 \mathrm{~nm}$ with an Argon laser

Figure 7 FOXO3 activation by AMPK is required for cell death. (a) CGNs were co-transfected with GFP and either the FOXO3 wt, DN-FOXO3 or FOXO3-6A expression plasmid. FOXO3 wt-transfected neurons treated with GLUT showed a significantly higher percentage of cell death ( $31.4 \pm 3.8 \%$ of total cells) than neurons exposed to vehicle $(6.4 \pm 2.7 \%) 16 \mathrm{~h}$ after GLUT/GLY $(100 / 10 \mu \mathrm{M}, 30 \mathrm{~min})$ exposure. However, cell death after the excitotoxic insult was negligible in cells transfected with DN-FOXO3 or FOXO3-6A, which presented a significant decrease in the percentage of cell death compared with $\mathrm{FOXO} 3$ wt-transfected neurons $\left({ }^{\star} P<0.05 ; n=3\right)$. (b) Cortical neurons were co-transfected with a GFP-expression vector and either AMPK siRNA, DN-FOXO3 or FOXO3-6A expression constructs. After $72 \mathrm{~h}$ transfection cultures were subjected to OGD $(30 \mathrm{~min})$ in a hypoxia chamber, and returned to normoxic/normoglycemic conditions. Cell death was determined $24 \mathrm{~h}$ later by evaluating the percentage of pyknotic nuclei in the GFP ${ }^{+}$ cells following Hoechst staining. Expression of either FOXO3-6A or DN-FOXO3 significantly reduced the percentage of neuronal cell death $(13.0 \pm 7.8 \%$ and $12.0 \pm 7.8 \%$ of total cells, respectively) compared with cells co-transfected with a control plasmid $(31.6 \pm 4.6 \%)\left({ }^{\star} P<0.05 ; n=4\right)$. Bar, $10 \mu \mathrm{M}$ 
(AOTF set to $0.5 \%$ ) and the emission collected in the range of $493-552 \mathrm{~nm}$. Hoechst 33258 was excited at $405 \mathrm{~nm}$ ( $1 \%$ transmission, ND filter and AOTF) and the emission detected in the range of 410 to $480 \mathrm{~nm}$. For the detection of plasma membrane permeabilization PI was excited at $543 \mathrm{~nm}$ (AOTF $1.5 \%$ transmission) and fluorescence emission was detected above $570 \mathrm{~nm}$. Images were captured at $5 \mathrm{~min}$ intervals at $1.5 \mu \mathrm{m}$ optical slice thickness. The resulting images were processed using MetaMorph 7.5 software (Molecular Devices, Wokingham, UK). The GFP average intensity fluorescence signal was quantified at the nucleus and cytoplasm to determine the nuclear/cytoplasmic FOXO3-GFP ratio. At least 33 single-cell experiments were analyzed for cells treated with GLUT, and 6 for those treated with experimental buffer. For quantification of $\mathrm{FOXO} 3$ nuclear translocation in neuronal populations, CGNs were plated on 24-well plates. At $3.5 \mathrm{~h}$ after GLUT/ experimental buffer exposure, neurons were incubated for $30 \mathrm{~min}$ with Hoechst 33258 (final concentration $1 \mu \mathrm{g} / \mathrm{ml}$ ) and PI (final concentration $0.2 \mu \mathrm{g} / \mathrm{ml}$ ). For each group, 80-90 cells were analyzed in three independent experiments using an Eclipse TE 2000s inverted microscope (Nikon, Amstelveen, The Netherlands) and a $40 \times 0.6$ NA objective. Images of GFP, PI and Hoechst signals of single neurons were taken and processed with Image $\mathrm{J}$ software $(\mathrm{NIH}$, Bethesda, MD, USA) to determine the nuclear/cytoplasmic FOXO3-GFP ratio. Neurons were scored as follows: FOXO3-GFP cytoplasmic (ratio $<0.9$ ), FOXO3-GFP nuclear (ratio $>1.1)$, FOXO3-GFP cytoplasmic/nuclear $(0.9<$ ratio $<1.1)$.

Western blotting and immunoprecipitation. Western blotting was performed as described. ${ }^{11}$ Neurons were lysed with PIK buffer (1\% NP-40, $150 \mathrm{mM} \mathrm{NaCl}, 20 \mathrm{mM}$ Tris, pH 7.4, $10 \%$ glycerol, $1 \mathrm{mM} \mathrm{CaCl}, 1 \mathrm{mM} \mathrm{MgCl}$, $400 \mu \mathrm{M}$ sodium vanadate, $0.2 \mathrm{mM}$ PMSF, $1 \mu \mathrm{g} / \mathrm{ml}$ leupeptin, $1 \mu \mathrm{g} / \mathrm{ml}$ aprotinin, $0.1 \%$ phosphatase inhibitor cocktails I and II of Sigma-Aldrich). Blots were probed with rabbit polyclonal antibodies to Bim (H-191) and HA (Y-11) from Santa Cruz Biotechnology (Dublin, Ireland). Phospho (Thr172) AMPK $\alpha$ antibody, total AMPK $\alpha$, phospho (Thr183/Thr185) JNK, total JNK, phospho (Ser473) AKT, total AKT, phospho (Ser63) c-Jun, phospho (Ser2448) mTOR total mTOR, phospho (Ser253) FOXO3 and phospho (Thr32) FOXO3 all from Cell Signaling and anti-Myc tag and total $\mathrm{FOXO3}$ antibodies from Millipore (Carrigtwohill, Ireland). Blots were also probed with mouse monoclonal antibodies to $\beta$-actin (clone DM 1A) and Anti-flag M2 from Sigma-Aldrich, and Neu-N and Anti-Phosphoserine clone 4A4 from Millipore. Horseradish peroxidase-conjugated secondary antibodies from Thermo Scientific (Fisher, Dublin, Ireland) were detected using SuperSignal West Pico Chemiluminescent Substrate (Thermo Scientific) and imaged using a FujiFilm LAS-3000 imaging system (FujiFilm, Dublin, Ireland). To normalize for protein load, membranes were reblotted (Re-Blot, Millipore) and incubated with an appropriate control antibody. Levels of the protein under study were expressed relative to protein load in each lane as determined by appropriate control protein content. pAMPK $\alpha$ levels were normalized to $\beta$-actin, as GLUT treatment decreased total AMPK levels. Densitometry analysis was performed using Image $\mathrm{J}$ software $(\mathrm{NIH})$. A representative blot is shown from a total of at least three independent experiments, except where indicated. Immunoprecipitation was performed in cultured neurons lysed in PIK buffer and centrifuged at $22000 \times g$ for $20 \mathrm{~min}$, and supernatants were incubated with antibodies bound to $25 \%$ agarose (Santa Cruz Biotechnology) overnight. The immunoprecipitates were washed three times with the same lysis buffer, resuspended in $2.5 \times$ SDS loading buffer and analyzed by western blot.

Cytosolic and nuclear fractionation. Nuclear and cytosolic fractions were obtained from total neuronal lysates as described. ${ }^{39}$ The quality of the fractionation was determined by assaying for the presence of the nuclear protein Neu-N.

Cell death assays. CGNs were cultured on 24-well plates. For determination of apoptotic morphology cells were stained live with Hoechst 33258 at a final concentration of $1 \mu \mathrm{g} / \mathrm{ml}$. After incubation for $10 \mathrm{~min}$, nuclear morphology was observed and cells with condensed nuclei were scored as pyknotic and expressed as a percentage of the total population. For determination of neuronal mortality cells were transfected with a pMax-GFP and different constructs under evaluation in a $1: 5$ ratio. GFP-positive cells (GFP ${ }^{+}$cells) were counted before treatment to determine the baseline level of transfected cells at time-point 0 , and were counted again $2 \mathrm{~h}$ after treatment. Results were expressed as percentage of $\mathrm{GFP}^{+}$cells at time 0 . Neurons were counted in 12 different fields using an Eclipse TE 300 inverted microscope (Nikon) and a $20 \times 0.45 \mathrm{NA}$ objective. All assays were done in triplicate in at least three independent experiments.
Luciferase assays. CGNs were transfected with luciferase reporter constructs containing different promoters along with a Renilla luciferase expression plasmid (pRL-TK) for normalization. Neurons were lysed in passive lysis buffer and luciferase activity was analyzed using a luminometer and dual luciferase assay kit according to the manufacturer Promega (Medical Supply, Dublin, Ireland). Transfections were performed in triplicate dishes and relative luciferase counts were normalized using TK Renilla luciferase co-transfection. Background luminescence was subtracted, and Luciferase activity was expressed as fold of the increase with respect to the control.

Immunocytochemistry. Following treatment, CGNs cultured on glass coverslips were fixed ( $3 \%$ paraformaldehyde), permeabilised $(0.1 \%$ Triton $x-100$ in PBS), blocked (5\% horse serum (Invitrogen Bio Sciences), $0.3 \%$ Triton $x-100$ in $\mathrm{PBS}$ ) and incubated with an antibody against $\mathrm{FOXO}$ (Millipore), at room temperature for $2 \mathrm{~h}$. FOXO3 staining was detected with an anti-rabbit AlexaFluor-568-conjugated secondary antibody (Invitrogen Bio Sciences). Nuclei were also stained with Hoechst 33258 . CGNs were imaged on the LSM 710 microscope detailed above and excited using 561, 488 and $405 \mathrm{~nm}$ lasers. Images were analyzed using ImageJ. The cytoplasmic and nuclear FOXO3 average intensity was quantified and CGNs were scored as cytoplasmic, nuclear or cyto/nuclear as described above.

Gene targeted mice. The generation and genotyping of bim - /- mice has been previously described. ${ }^{40}$ The bim $-/-$ mice were originally generated on a mixed C57BL/6 $\times 129$ SV genetic background, using 129SV-derived ES cells, but had been backcrossed for $>12$ generations onto the $\mathrm{C} 57 \mathrm{BL} / 6$ background.

Oxygen and glucose deprivation in cortical neurons. Cortical neurons cultured on 24-well plates were transfected with a pMax-GFP plasmid and different constructs under evaluation in a $1: 5$ ratio. Before $O G D$ induction, cells were rinsed in pre-warmed glucose-free media and transferred to the anaerobic chamber. The anaerobic chamber had an atmosphere consisting of $1.5 \% \mathrm{O}_{2}, 5 \%$ $\mathrm{CO}_{2}$ and $85 \% \mathrm{~N}_{2}$. Cells were incubated in OGD media consisting of $2 \mathrm{mM} \mathrm{CaCl}_{2}$, $125 \mathrm{mM} \mathrm{NaCl}, 25 \mathrm{mM} \mathrm{NaHCO}_{3}, 2.5 \mathrm{mM} \mathrm{KCl}, 1.25 \mathrm{mM} \mathrm{NaH}_{2} \mathrm{PO}_{4}, 2 \mathrm{mM} \mathrm{MgSO}_{4}$ and $10 \mathrm{mM}$ Sucrose and had a pH of 7.4. After $30 \mathrm{~min}$ of OGD, cells were returned to normoxic conditions containing $21 \% \mathrm{O}_{2}$ and $5 \% \mathrm{CO}_{2}$. Control cells remained in normoxic conditions throughout. After $24 \mathrm{~h}$ cells were stained live with Hoechst 33258 and cell death assessed as described above.

Computational modeling. We modeled the AMPK-dependent FOXO3 activation by translating the reaction networks shown in Figure 5 a into Ordinary Differential Equations. As model input, different activation profiles of AMPK (pAMPK) were resembled by a Gaussian curve with a maximum of $270 \mathrm{nM}$ at a reference time-point and full-width-half-maxima between 0 and 150 min to mimic transient and prolonged activation. In a first activation step, phosphorylated AMPK (pAMPK) was assumed to inhibit mTOR and AKT activity, leading to dephosphorylation of $\mathrm{FOXO3} \mathrm{(FOXO3} 3_{\text {dephos}}$ ). In a second activation step (Figure 5a (2)), pAMPK directly phosphorylated $\mathrm{FOXO}_{\text {dephos }}$ to a fraction (FOXO3 ${ }_{\text {AMPK }}$ ) assumed to be capable of inducing bim transcription. The reaction network is depicted in Supplementary Table 1. Protein-protein interactions, including all phosphorylation and dephosphorylation events, were modeled by mass action kinetics, whereas a Hill kinetic was assumed for bim transcription. First-order degradation kinetics were assumed for dephosphorylated proteins. Phosphorylated protein fractions were assumed to recover to a steady-state level. The complete Matlab code is available by request from the authors.

Statistical analysis. Data are expressed as mean \pm S.D. Differences among groups were analyzed by one-way ANOVA followed by Tukey test. Comparison between two groups was performed with the $t$ test. $P<0.05$ was considered significant.

\section{Conflict of Interest}

The authors declare no conflict of interest.

Acknowledgements. This study was supported by Marie Curie IEF (PIEF-GA-2009-237765), Science Foundation Ireland (08/ IN1/ 1949), and the Health Research Board in Ireland (PHD/2007/11). We thank Dr. Hans Georg Koenig for discussions and support, Ina Woods and Sarah Cannon for excellent 
technical assistance, and Andreas Strasser (WEHI, Melbourne, Australia) for bim-deficient mice.

1. Van Den Bosch L, Van Damme P, Bogaert E, Robberecht W. The role of excitotoxicity in the pathogenesis of amyotrophic lateral sclerosis. Biochim Biophys Acta 2006; 1762: 1068-1082.

2. Bezprozvanny I, Mattson MP. Neuronal calcium mishandling and the pathogenesis of Alzheimer's disease. Trends Neurosci 2008; 31: 454-463.

3. Lee JM, Zipfel GJ, Choi DW. The changing landscape of ischaemic brain injury mechanisms. Nature 1999; 399(6738 Suppl): A7-A14.

4. Sattler R, Tymianski M. Molecular mechanisms of glutamate receptor-mediated excitotoxic neuronal cell death. Mol Neurobiol 2001; 24: 107-129.

5. Atlante A, Gagliardi S, Minervini GM, Marra E, Passarella S, Calissano P. Rapid uncoupling of oxidative phosphorylation accompanies glutamate toxicity in rat cerebellar granule cells. Neuroreport 1996; 7: 2519-2523.

6. Nicholls DG. Oxidative stress and energy crises in neuronal dysfunction. Ann NY Acad Sci 2008; 1147: 53-60.

7. Ward MW, Rego AC, Frenguelli BG, Nicholls DG. Mitochondrial membrane potential and glutamate excitotoxicity in cultured cerebellar granule cells. J Neurosci 2000; 20: 7208-7219.

8. Ward MW, Huber HJ, Weisova P, Dussmann H, Nicholls DG, Prehn JH. Mitochondrial and plasma membrane potential of cultured cerebellar neurons during glutamate-induced necrosis, apoptosis, and tolerance. J Neurosci 2007; 27: 8238-8249.

9. Weisova P, Davila D, Tuffy LP, Ward MW, Concannon CG, Prehn JH. Role of 5'-Adenosine monophosphate-activated protein kinase in cell survival and death responses in neurons. Antioxid Redox Signal 2011; 14: 1863-1876.

10. Hardie DG. The AMP-activated protein kinase pathway-new players upstream and downstream. J Cell Sci 2004; 117(Pt 23): 5479-5487.

11. Weisova $\mathrm{P}$, Concannon CG, Devocelle M, Prehn JH, Ward MW. Regulation of glucose transporter 3 surface expression by the AMP-activated protein kinase mediates tolerance to glutamate excitation in neurons. J Neurosci 2009; 29: 2997-3008.

12. Li J, McCullough LD. Effects of AMP-activated protein kinase in cerebral ischemia. J Cereb Blood Flow Metab 2010; 30: 480-492.

13. Concannon CG, Tuffy LP, Weisova P, Bonner HP, Davila D, Bonner $C$ et al. AMP kinasemediated activation of the BH3-only protein Bim couples energy depletion to stressinduced apoptosis. J Cell Biol 2010; 189: 83-94.

14. Li J, Zeng Z, Viollet B, Ronnett GV, McCullough LD. Neuroprotective effects of adenosine monophosphate-activated protein kinase inhibition and gene deletion in stroke. Stroke 2007; 38: 2992-2999.

15. Leist M, Jaattela M. Four deaths and a funeral: from caspases to alternative mechanisms. Nat Rev Mol Cell Biol 2001; 2: 589-598.

16. Gilley J, Coffer PJ, Ham J. FOXO transcription factors directly activate bim gene expression and promote apoptosis in sympathetic neurons. J Cell Biol 2003; 162: 613-622.

17. Davila $D$, Torres-Aleman I. Neuronal death by oxidative stress involves activation of FOXO3 through a two-arm pathway that activates stress kinases and attenuates insulinlike growth factor I signaling. Mol Biol Cell 2008; 19: 2014-2025.

18. Biswas SC, Shi Y, Sproul A, Greene LA. Pro-apoptotic Bim induction in response to nerve growth factor deprivation requires simultaneous activation of three different death signaling pathways. J Biol Chem 2007; 282: 29368-29374.

19. Bolster DR, Crozier SJ, Kimball SR, Jefferson LS. AMP-activated protein kinase suppresses protein synthesis in rat skeletal muscle through down-regulated mammalian target of rapamycin (mTOR) signaling. J Biol Chem 2002; 277: 23977-23980.

20. Dormond O, Madsen JC, Briscoe DM. The effects of mTOR-Akt interactions on antiapoptotic signaling in vascular endothelial cells. J Biol Chem 2007; 282: 23679-23686.

21. Brunet A, Bonni A, Zigmond MJ, Lin MZ, Juo P, Hu LS et al. Akt promotes cell survival by phosphorylating and inhibiting a Forkhead transcription factor. Cell 1999; 96: 857-868.
22. Essers MA, Weijzen S, de Vries-Smits AM, Saarloos I, de Ruiter ND, Bos JL et al. FOXO transcription factor activation by oxidative stress mediated by the small GTPase Ral and JNK. EMBO J 2004; 23: 4802-4812.

23. Culmsee C, Monnig J, Kemp BE, Mattson MP. AMP-activated protein kinase is highly expressed in neurons in the developing rat brain and promotes neuronal survival following glucose deprivation. J Mol Neurosci 2001; 17: 45-58.

24. Greer EL, Oskoui PR, Banko MR, Maniar JM, Gygi MP, Gygi SP et al. The energy sensor AMP-activated protein kinase directly regulates the mammalian $\mathrm{FOXO3}$ transcription factor. J Biol Chem 2007; 282: 30107-30119.

25. Inoue E, Yamauchi J. AMP-activated protein kinase regulates PEPCK gene expression by direct phosphorylation of a novel zinc finger transcription factor. Biochem Biophys Res Commun 2006; 351: 793-799.

26. Gundewar S, Calvert JW, Jha S, Toedt-Pingel I, Ji SY, Nunez D et al. Activation of AMPactivated protein kinase by metformin improves left ventricular function and survival in heart failure. Circ Res 2009; 104: 403-411.

27. Meisse D, Van de Casteele M, Beauloye C, Hainault I, Kefas BA, Rider MH et al. Sustained activation of AMP-activated protein kinase induces c-Jun N-terminal kinase activation and apoptosis in liver cells. FEBS Lett 2002; 526: 38-42.

28. Cai Y, Martens GA, Hinke SA, Heimberg H, Pipeleers D, Van de Casteele M. Increased oxygen radical formation and mitochondrial dysfunction mediate beta cell apoptosis under conditions of AMP-activated protein kinase stimulation. Free Radic Biol Med 2007; 42: 64-78.

29. Kilbride SM, Farrelly AM, Bonner C, Ward MW, Nyhan KC, Concannon CG et al. AMPactivated protein kinase mediates apoptosis in response to bioenergetic stress through activation of the pro-apoptotic Bcl-2 homology domain-3-only protein BMF. J Biol Chem 2010; 285: 36199-36206.

30. Song G, Ouyang G, Bao $\mathrm{S}$. The activation of Akt/PKB signaling pathway and cell survival. $J$ Cell Mol Med 2005; 9: 59-71.

31. Millward TA, Zolnierowicz S, Hemmings BA. Regulation of protein kinase cascades by protein phosphatase 2A. Trends Biochem Sci 1999; 24: 186-191.

32. Stambolic V, Suzuki A, de la Pompa JL, Brothers GM, Mirtsos C, Sasaki T et al. Negative regulation of PKB/Akt-dependent cell survival by the tumor suppressor PTEN. Cell 1998, 95: 29-39.

33. Mangan S, Alon U. Structure and function of the feed-forward loop network motif. Proc Natl Acad Sci USA 2003; 100: 11980-11985.

34. Shen-Orr SS, Milo R, Mangan S, Alon U. Network motifs in the transcriptional regulation network of Escherichia coli. Nat Genet 2002; 31: 64-68.

35. Li D, Qu Y, Mao M, Zhang X, Li J, Ferriero D et al. Involvement of the PTEN-AKT-FOXO3a pathway in neuronal apoptosis in developing rat brain after hypoxia-ischemia. J Cereb Blood Flow Metab 2009; 29: 1903-1913.

36. Coffey ET, Smiciene G, Hongisto V, Cao J, Brecht S, Herdegen T et al. c-Jun N-terminal protein kinase (JNK) $2 / 3$ is specifically activated by stress, mediating c-Jun activation, in the presence of constitutive JNK1 activity in cerebellar neurons. J Neurosci 2002; 22. 4335-4345.

37. Brunet A, Sweeney LB, Sturgill JF, Chua KF, Greer PL, Lin Y et al. Stress-dependent regulation of FOXO transcription factors by the SIRT1 deacetylase. Science 2004; 303: 2011-2015.

38. Ju TC, Chen HM, Lin JT, Chang CP, Chang WC, Kang JJ et al. Nuclear translocation of AMPK-\{alpha\}1 potentiates striatal neurodegeneration in Huntington's disease. J Cell Biol 2011; 194: 209-227.

39. Essafi A, Gomes AR, Pomeranz KM, Zwolinska AK, Varshochi R, McGovern UB et al. Studying the subcellular localization and DNA-binding activity of FoxO transcription factors, downstream effectors of PI3K/Akt. Methods Mol Biol 2009; 462: 201-211.

40. Bouillet P, Metcalf D, Huang DC, Tarlinton DM, Kay TW, Kontgen F et al. Proapoptotic Bc2 relative Bim required for certain apoptotic responses, leukocyte homeostasis, and to preclude autoimmunity. Science 1999; 286: 1735-1738.

\section{Supplementary Information accompanies the paper on Cell Death and Differentiation website (http://www.nature.com/cdd)}

